OPEN ACCESS

Edited by:

Brian Godman, Karolinska Institutet (KI),

Sweden

Reviewed by:

Christel Hanson,

Tshwane University of

Technology, South Africa

Andriette Van Jaarsveld,

Mediclinic Southern Africa,

South Africa

*Correspondence:

Chong Guo

guochongghn@163.com

Specialty section:

This article was submitted to Pharmaceutical Medicine and

Outcomes Research,

a section of the journal

Frontiers in Pharmacology

Received: 06 May 2019 Accepted: 11 December 2019

Published: 15 January 2020

Citation:

Gong $Q-Q, X u Y-Q, X u J$, Ding $X-Y$ and Guo C (2020) Meta-Analysis of Randomized Controlled Trials Using Botulinum Toxin A at Different Dosages for Urinary Incontinence in Patients With Overactive Bladder.

Front. Pharmacol. 10:1618. doi: 10.3389/fphar.2019.01618

\section{Meta-Analysis of Randomized Controlled Trials Using Botulinum Toxin A at Different Dosages for Urinary Incontinence in Patients With Overactive Bladder}

\author{
Qin-Qin Gong ${ }^{1}$, Yu-Qiong $X u^{2}$, Jun $X_{u^{3}}$, Xiao-Yan Ding ${ }^{3}$ and Chong Guo ${ }^{1,3 *}$ \\ ${ }^{1}$ Center for Women's Healthcare Sciences, Taihe Hospital, Hubei University of Medicine, Shiyan, China, ${ }^{2}$ Department of \\ Gynaecology, Peking University Shenzhen Hospital, Shenzhen, China, ${ }^{3}$ Center for Gynaecology and Obstetrics, Taihe \\ Hospital, Hubei University of Medicine, Shiyan, China
}

Background: Urinary incontinence $(\mathrm{UI})$ is a common and refractory complication for patients with neurogenic detrusor overactivity (NDO) or idiopathic overactive bladder (IOAB).

Objectives: To evaluate the effect of Botulinum toxin A (BTX-A) based on different dosages strategy for UI.

Method: The MEDLINE, Ovid EMbase, The Cochrane Central Register of Controlled Trials (CENTRAL), China National Knowledge Internet (CNKI), and WanFang database were searched for relevant published randomized controlled trials (RCTs) between 1969 to September 31, 2018. All database were searched to identify relevant randomized controlled trials (RCTs) that investigated the clinical benefit of BTX-A for management of UI in patients with NDO and IOAB.

Results: This meta-analysis involved 19 original studies. The BTX-A was superior to placebo in reducing episodes of $\mathrm{UI}$ for NDO patients in all subgroups of different dosages for different durations, and also reduced maximum detrusor pressure in all kinds of $200 \mathrm{U}$ and $300 \mathrm{U}$ at 6 weeks. However, it increased post void residual in different dosages of $200 U$ at 2 weeks. For IOAB patients, compared to placebo, BTX-A increased detrusor compliance for different dosages of $200 \mathrm{U}$ and $300 \mathrm{U}$ at 12 and 36 weeks, but it increased risk of urinary tract infections at other dosages.

Conclusions: This meta-analysis indicated that BTX-A 200U and $300 \mathrm{U}$ are more effective than placebo in the treatment of NDO, with minimal, local, and manageable adverse events. Furthermore, BTX-A $300 \mathrm{U}$ and $200 \mathrm{U}$ could also improve detrusor compliance of IOAB. However, more RCTs would still be necessary to explore the effect of BTX-A on management of $\mathrm{UI}$ in NDO and IOAB patients.

Keywords: urinary incontinence, neurogenic detrusor overactivity, idiopathic overactive bladder, Botulinum toxin A, meta-analysis 


\section{INTRODUCTION}

Overactive bladder (OAB) is defined as a series of symptoms (Abrams et al., 2002; White \& Iglesia, 2016), including urinary urgency (usually accompanied by frequency and nocturia) and urinary incontinence (UI) in the absence of urinary tract infection (UTI) or other obvious pathology, according to the statement established by International Continence Society. The classification of OAB (Stewart et al., 2003; Lawrence et al., 2008) are generally considered two types: neurogenic detrusor overactivity (NDO) and idiopathic overactive bladder (IOAB). Almost 16.9\% women suffer UI caused by $\mathrm{OAB}$ in the United States, which means that this disease has become a considerable common health issue with significant effects on women mentally and physically, of which mainly on account of urgency UI (Durden and Walker, 2018).

For management of $\mathrm{UI}$ in $\mathrm{OAB}$ patients, anticholinergic medicine is currently recommended as the first-line therapy (Garely and Burrows, 2002). Nevertheless, the anticholinergic medicine is increasingly inappropriate for long-term therapy of $\mathrm{NDO}$ and IOAB, which is reflected in the unsatisfied effect and potential complications such as vesicoureteral reflux and even renal failure (Majumdar, 2004; Asimakopoulos et al., 2012), and also the high socioeconomic cost is considerable. Therefore, metaanalyses (Cui et al., 2013; Mehta et al., 2013; Cui et al., 2015; Sun et al., 2015; Zhang et al., 2015; Zhou et al., 2015; Cheng et al., 2016; $\mathrm{Gu}$ et al., 2017) evaluating the therapeutic effect of botulinum toxin A (BTX-A) on UI in OAB patients have increased as well as the relevant RCTs in recent years, in which the BTX-A demonstrated a satisfied clinical benefit. (Flynn et al., 2004; Kessler et al., 2005; Kuo, 2005; Popat et al., 2005; Rajkumar et al., 2005; Schulte-Baukloh et al., 2005; Schmid et al., 2006) Furthermore, the BTX-A is recommended for management of UI in $\mathrm{OAB}$ patient by the American Urological Association (AUA) guidelines (Gormley et al., 2015), and the other interventions consist of education and behavior therapies. Nevertheless, the effect and safety of BTX-A is still controversial, furthermore, the clinical outcomes by different dosages also remains blank.

Therefore, we performed the systemic review and meta-analysis to evaluate the effect and safety of BTX-A at different dosages for the management of UI in patients with NDO and IOAB.

\section{METHODS}

This systemic review and meta-analysis was conducted in accordance with the Preferred Reporting Items for Systematic Reviews and Meta-Analyses guidelines (PRISMA) (Moher et al., 2009) and Cochrane Collaboration's systematic review framework (Higgins JP, 2011).

\section{SEARCH STRATEGY}

The Ovid MEDLINE, Ovid EMbase, and the Cochrane Central Register of Controlled Trials (CENTRAL), China National Knowledge Internet (CNKI), and WanFang databases were searched between 1964 to September 31, 2018. All database were searched to identify relevant randomized controlled trials (RCTs) that investigated the clinical benefit of BTX-A for management of UI in patients with NDO and IOAB. All search strategy is described in Supplementary Method 1.

\section{INCLUSION CRITERIA AND EXCLUSION CRITERIA}

RCTs were identified if the following criteria were met: (1) the patients with NDO or IOAB were confirmed; (2) patients $>18$ year; (3) studies compared BTX-A with placebo or BTX-A at different dosages, which reported in English and Chinese.

Studies were excluded for the following reasons: (1) stress incontinence; (2) duplicate studies; (3) for continuous outcomes, the standard deviations (SD) was still missing after contacting with the authors; (4) evaluated the clinical benefit of different injection sites only; (5) follow-up period was less than 1 week.

\section{DATA EXTRACTION}

After independently reviewing the included studies by two reviewers (Hui-Yun $\mathrm{Gu}$ and Shuang Li), all following information were extracted: (1) first author, published year, regions, number of female patients, mean age, etiology of UI, and other basic disease; (2) the events, mean and SD of outcomes; (3) the effect outcomes and events in different time periods (2 weeks, 6 weeks, and 12 weeks) including UI episodes per week, maximum detrusor pressure (MDP), detrusor compliance (DC), and post void residual (PVR). The adverse events including urinary tract infections (UTI), urinary retention, hematuria, muscle weakness, and PVR-related catheterization were only recorded the events after follow-up without separately extracted according to different periods. The outcomes were expressed as two observation periods: short-term ( $\leq 12$ weeks) and long-term ( $>12$ weeks).

\section{QUALITY OF INCLUDED STUDIES AND RISK OF BIAS}

For evaluating the risk of bias in RCTs, the Cochrane Collaboration's tool (Higgins et al., 2011) was performed by two independent reviewers (Hui-Yun Gu and Shuang Li), which considers seven domains including adequacy of blinding of participants, sequence generation, allocation concealment, blinding of outcome assessment, selective outcome reporting, incomplete outcome data, and other potential sources of bias, in each item was graded as "high risk", "low risk", or "unclear".

\section{STATISTICAL ANALYSIS}

Dichotomous outcomes were expressed as the relative risk (RR) with 95\% confidence interval (CI) (Deeks, 2002; Higgins JP, 2011), and continuous outcomes were expressed as mean 
difference (MD) (Higgins JP, 2011) with 95\% CI. Both of them was bounded by $\mathrm{P}<0.05$ for statistical significance. Forest plot was carried out to summarize the outcomes. Heterogeneity was tested using I2 tests (Melsen et al., 2014), in which the significance level was set to $\mathrm{P}<0.1$. $\mathrm{I}^{2}$ statistic provides an estimate of the percentage of inconsistency thought to be due to chance (Higgins JP, 2002). Initial analyses were performed using a fixed-effects model when $\mathrm{I}^{2}<40 \%$, the random model was performed when $\mathrm{I}^{2}>40 \%$.

The different dosages and types of IOAB and NDO were performed by subgroup analyses. All statistical analyses were performed using the Stata software (Versions, 12.0).

\section{RESULTS}

\section{Characteristics and Risk of Bias of Eligible Studies}

A total of 527 RCTs were initially identified, in which 86 studies were eliminated due to duplicates, then 441 studies were excluded in preliminary screening, therefore, only 53 articles were independently read in full text filtering. Ultimately, 34 studies were excluded (Supplementary Method 2), and a total of 19 articles (Schurch et al., 2005; Sahai et al., 2007; Brubaker et al., 2008; Cohen et al., 2009; Dmochowski et al., 2010; Altaweel et al., 2011; Cruz et al., 2011; Herschorn et al., 2011; Rovner et al., 2011; Denys et al., 2012; Ginsberg et al., 2012; Chapple et al., 2013; Ginsberg et al., 2013; Kennelly et al., 2013; Nitti et al., 2013; Rovner et al., 2013; Chen \& Kuo, 2014; Sievert et al., 2014; Abdelwahab et al., 2015) were included in this meta-analysis (Figure 1).

Overall, 19 RCTs (Schurch et al., 2005; Sahai et al., 2007; Brubaker et al., 2008; Cohen et al., 2009; Dmochowski et al., 2010; Altaweel et al., 2011; Cruz et al., 2011; Herschorn et al., 2011; Rovner et al., 2011; Denys et al., 2012; Ginsberg et al., 2012; Chapple et al., 2013; Ginsberg et al., 2013; Kennelly et al., 2013; Nitti et al., 2013; Rovner et al., 2013; Chen \& Kuo, 2014; Sievert et al., 2014; Abdelwahab et al., 2015) comprised 5,596 participants who were diagnosed as IOAB or NDO with UI were included in this study. Eight RCTs with 2,097 patients in $\mathrm{NDO}$, and the remaining 11 RCTs comprised 3,499 IOAB
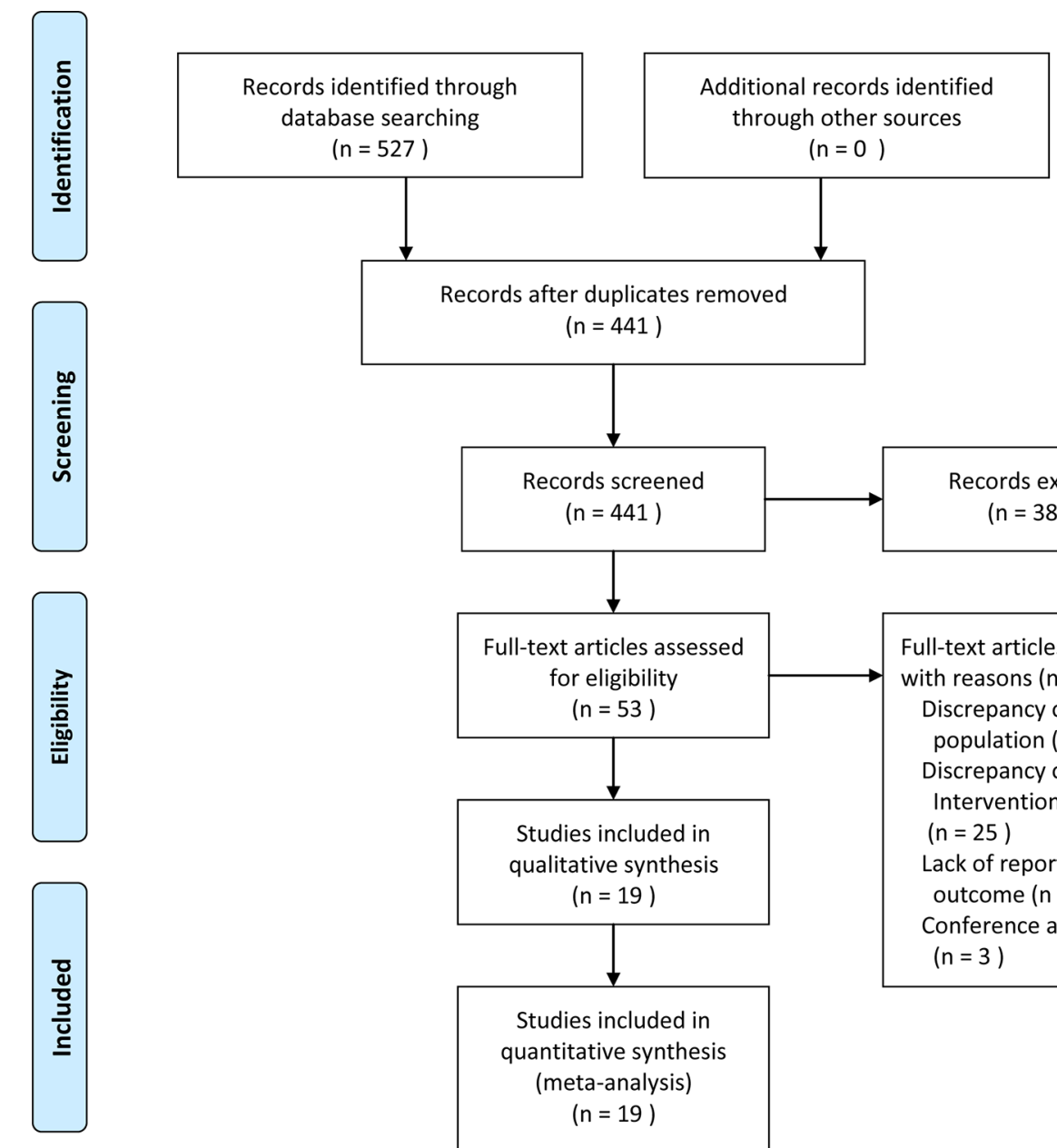

Records after duplicates removed $(n=441)$

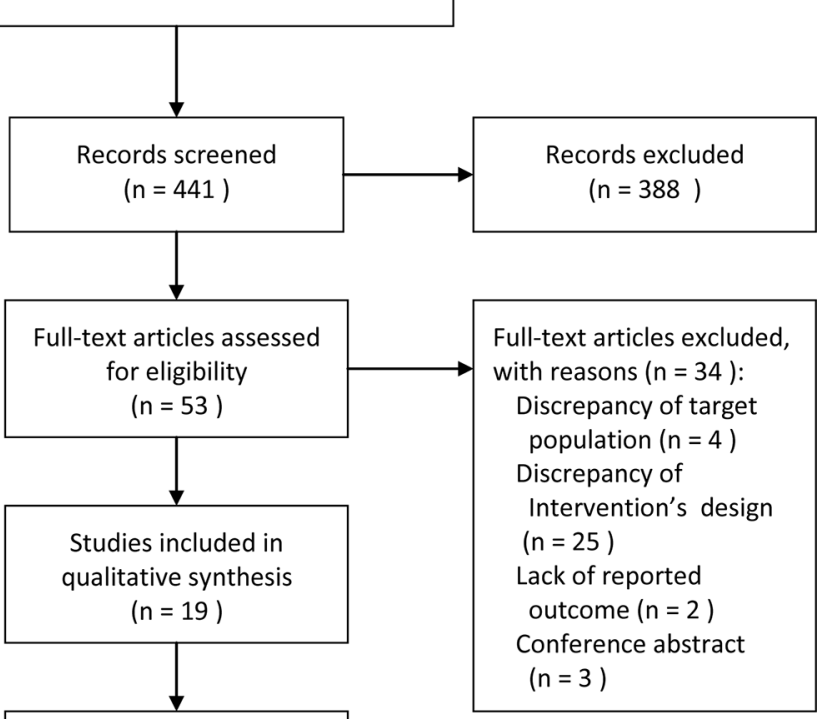

Studies included in quantitative synthesis (meta-analysis) $(n=19)$

FIGURE 1 | A flow diagram of the study selection process. 
patients were treated with one of these two interventions: BTX-A or placebo. The characteristics of included studies were presented in Table 1.

The risk of bias in eligible studies was assessed by two independent authors (Hui-Yun Gu and Shuang $\mathrm{Li}$ ) using the Cochrane Collaboration tool, and found that the 19 included RCTs were all graded as high-quality studies (Table 2 ).

\section{Outcomes}

All the results of following outcomes for $\mathrm{NDO}$ and IOAB patients were shown in Table 3 and Table 4, respectively.

\section{Effectiveness}

\section{Episodes of Urinary Incontinence (UI) Per Week}

For NDO in Short-Term Observation Period

At 2 weeks. Only one study (Cruz et al., 2011) reported this outcome, and the BTX-A (200U) showed a significant improvement in reducing episodes of UI per week $(\mathrm{MD}=$ -9.10, 95\% CI: $-14.10,-4.10)$ compared to placebo. However, no significant effect was obtained in BTX-A (300U) compared to placebo $(\mathrm{MD}=-6.10,95 \% \mathrm{CI}:-12.54,0.34)$. In addition, no significant effect between BTX-A at $300 \mathrm{U}$ and $200 \mathrm{U}$ was observed ( $\mathrm{MD}=3.00,95 \% \mathrm{CI}:-3.30,9.30)$ (Figure 2).

TABLE 1 | Characteristics of individual study.

\begin{tabular}{|c|c|c|c|c|c|c|c|c|c|}
\hline Study & Year & Region & $\begin{array}{l}\text { No. of } \\
\text { patients } \\
\text { (female) }\end{array}$ & $\begin{array}{c}\text { Ages } \\
\text { Mean(SD) }\end{array}$ & Design & $\begin{array}{l}\text { Classification } \\
\text { of urinary } \\
\text { incontinence }\end{array}$ & $\begin{array}{c}\text { Basic } \\
\text { diseases }\end{array}$ & Intervention & $\begin{array}{l}\text { Follow- } \\
\text { up } \\
\text { (weeks) }\end{array}$ \\
\hline Schurch & 2005 & Switzerland & $59(23)$ & 41 & $\begin{array}{l}\text { Randomized, } \\
\text { doubled-blind }\end{array}$ & NDO & $\begin{array}{l}\text { MS:6, } \\
\text { SCl:53 }\end{array}$ & $\begin{array}{l}\text { Group 1: BTX-A 300U (19); Group 2: BTX-A } 200 U \\
\text { (19); Group 3: Placebo (21) }\end{array}$ & 6 \\
\hline Sahai & 2007 & UK & $34(19)$ & $49.8,50.8$ & $\begin{array}{l}\text { Randomized, } \\
\text { doubled-blind }\end{array}$ & IOAB & NA & Group 1: BTX-A $200 U$ (16); Group 2: Placebo (18) & NA \\
\hline Brubaker & 2008 & USA & 43 & $\begin{array}{l}64.7(14.5) \\
69.2(13.5)\end{array}$ & $\begin{array}{l}\text { Randomized, } \\
\text { doubled-blind }\end{array}$ & IOAB & NA & Group 1: BTX-A $200 U$ (28); Group 2: Placebo (15) & NA \\
\hline Cohen & 2009 & USA & 44 & NA & $\begin{array}{l}\text { Randomized, } \\
\text { doubled-blind }\end{array}$ & IOAB & NA & $\begin{array}{l}\text { Group 1: BTX-A 150U (22); Group 2: BTX-A } 100 U \\
\text { (22) }\end{array}$ & NA \\
\hline Dmochowski & 2010 & USA & 313(288) & 58.8 & $\begin{array}{l}\text { Randomized, } \\
\text { doubled-blind }\end{array}$ & IOAB & NA & $\begin{array}{l}\text { Group 1: BTX-A 50U (56); Group 2: BTX-A } 100 U \\
\text { (55); Group 3: BTX-A 150U (50); Group 4: BTX-A } \\
\text { 200U (52); Group 5: BTX-A 300U (55); Group 6: } \\
\text { Placebo (43) }\end{array}$ & NA \\
\hline Rovner & 2011 & USA & $313(288)$ & 58.8 & $\begin{array}{l}\text { Randomized, } \\
\text { doubled-blind }\end{array}$ & $\mathrm{IOAB}$ & NA & $\begin{array}{l}\text { Group 1: BTX-A 50U (57); Group 2: BTX-A 100U } \\
\text { (54); Group 3: BTX-A 150U (49); Group 4: BTX-A } \\
\text { 200U (53); Group 5: BTX-A 300U (56); Group 6: } \\
\text { Placebo (44) }\end{array}$ & 12,36 \\
\hline Cruz & 2011 & Portugal & $275(155)$ & $\begin{array}{c}46(13.1) \\
44.4(13.9) \\
46.9(13.4)\end{array}$ & $\begin{array}{l}\text { Randomized, } \\
\text { doubled-blind }\end{array}$ & NDO & $\begin{array}{l}\text { MS: } 154 \\
\text { SCl: } 121\end{array}$ & $\begin{array}{l}\text { Group 1: BTX-A } 200 U \text { (92); Group 2: BTX-A } 300 U \\
\text { (91); Group 3: Placebo (92) }\end{array}$ & $2,6,12$ \\
\hline Herschorn & 2011 & Canada & $57(23)$ & 42.8 & $\begin{array}{l}\text { Randomized, } \\
\text { doubled-blind }\end{array}$ & NDO & $\begin{array}{l}\text { MS:19, } \\
\text { SCl:38 }\end{array}$ & Group 1: BTX-A 300U (28); Group 2: Placebo (29) & NA \\
\hline Altaweel & 2011 & $\begin{array}{l}\text { Saudi } \\
\text { Arabia }\end{array}$ & 22 & NA & $\begin{array}{l}\text { Randomized, } \\
\text { doubled-blind }\end{array}$ & IOAB & NA & $\begin{array}{l}\text { Group 1: BTX-A } 200 U \text { (11); Group 2: BTX-A } 100 U \\
\text { (11) }\end{array}$ & NA \\
\hline Denys & 2012 & France & 199(87) & $62.3,61.7$ & $\begin{array}{l}\text { Randomized, } \\
\text { doubled-blind }\end{array}$ & IOAB & NA & $\begin{array}{l}\text { Group 1: BTX-A 50U (23); Group 2: BTX-A } 100 U \\
\text { (23); Group 3: BTX-A 150U (30); Group 4: Placebo } \\
\text { (31) }\end{array}$ & 12 \\
\hline Ginsberg & 2012 & USA & $416(245)$ & $46(13)$ & $\begin{array}{l}\text { Randomized, } \\
\text { doubled-blind }\end{array}$ & NDO & $\begin{array}{l}\text { MS:227, } \\
\text { SCl:189 }\end{array}$ & $\begin{array}{l}\text { Group 1: BTX-A } 200 U \text { (135); Group 2: BTX-A } 300 U \\
\text { (127); Group 3: Placebo (145) }\end{array}$ & 6 \\
\hline Rovner & 2013 & USA & $691(400)$ & $\begin{array}{c}45.9 \\
45.6,46.2\end{array}$ & $\begin{array}{l}\text { Randomized, } \\
\text { doubled-blind }\end{array}$ & NDO & $\begin{array}{l}\text { MS: } 103 \\
\text { SCl:138 }\end{array}$ & $\begin{array}{l}\text { Group 1: BTX-A } 200 U \text { (227); Group 2: BTX-A } 300 U \\
\text { (223); Group 3: Placebo (241) }\end{array}$ & 6 \\
\hline Nitti & 2013 & USA & $557(497)$ & $\begin{array}{c}61.7(12.7) \\
61(13.1)\end{array}$ & $\begin{array}{l}\text { Randomized, } \\
\text { doubled-blind }\end{array}$ & IOAB & NA & $\begin{array}{l}\text { Group 1: BTX-A 100U (278); Group 2: Placebo } \\
(272)\end{array}$ & 12 \\
\hline Kennelly & 2013 & USA & $387(233)$ & 46.4 & $\begin{array}{l}\text { Randomized, } \\
\text { doubled-blind }\end{array}$ & NDO & $\mathrm{SCl}, \mathrm{MS}$ & $\begin{array}{l}\text { Group 1: BTX-A } 300 U \text { (185); Group 2: BTX-A } 200 U \\
\text { (202) }\end{array}$ & 6 \\
\hline Ginsberg & 2013 & USA & $381(311)$ & $\begin{array}{l}49.7(12.1) \\
49.9(10.7) \\
50.2(10.7)\end{array}$ & $\begin{array}{l}\text { Randomized, } \\
\text { doubled-blind }\end{array}$ & NDO & $\begin{array}{l}\text { MS:381, } \\
\text { SCl:310 }\end{array}$ & $\begin{array}{l}\text { Group 1: BTX-A 200U(227); Group 2: BTX-A } 300 U \\
\text { (223); Group 3: Placebo (241) }\end{array}$ & 6 \\
\hline Chapple & 2013 & UK & $548(473)$ & $\begin{array}{l}59.5(15.5) \\
59.2(14.1)\end{array}$ & $\begin{array}{l}\text { Randomized, } \\
\text { doubled-blind }\end{array}$ & IOAB & NA & $\begin{array}{l}\text { Group 1: BTX-A 100U (277); Group 2: Placebo } \\
\text { (271) }\end{array}$ & NA \\
\hline Sievert & 2014 & Germany & $\begin{array}{l}1105 \\
(970)\end{array}$ & $\begin{array}{l}60.6(14.2) \\
60.1(13.6)\end{array}$ & $\begin{array}{l}\text { Randomized, } \\
\text { doubled-blind }\end{array}$ & IOAB & NA & $\begin{array}{l}\text { Group 1: BTX-A 100U (557); Group 2: Placebo } \\
(548)\end{array}$ & NA \\
\hline Chen & 2014 & Taiwan & $72(29)$ & 41.5 & $\begin{array}{l}\text { Randomized, } \\
\text { doubled-blind }\end{array}$ & NDO & $\mathrm{SCl}$ & $\begin{array}{l}\text { Group 1: BTX-A 300U (34); Group 2: BTX-A } 200 U \\
\text { (38) }\end{array}$ & NA \\
\hline Abdelwahab & 2015 & Egypt & $80(63)$ & $\begin{array}{l}31.35 \\
30.22\end{array}$ & $\begin{array}{l}\text { Randomized, } \\
\text { doubled-blind }\end{array}$ & IOAB & NA & $\begin{array}{l}\text { Group 1: BTX-A 100U (40); Group 2: BTX-A } 200 U \\
\text { (40) }\end{array}$ & 12,36 \\
\hline
\end{tabular}

BTX-A, Botulinum toxin A; NDO, Neurogenic detrusor overactivity; IOAB, Idiopathic overactive bladder; MS, Multiple sclerosis; SCI, Spinal cord injury; NA, Not available. 
TABLE 2 | The risk of bias in the included studies.

\begin{tabular}{|c|c|c|c|c|c|c|c|c|}
\hline Authors & Year & $\begin{array}{c}\text { Random sequence } \\
\text { generation }\end{array}$ & $\begin{array}{l}\text { Allocation con- } \\
\text { cealment }\end{array}$ & $\begin{array}{c}\text { Blinding of participants } \\
\text { and personnel }\end{array}$ & $\begin{array}{c}\text { Blinding of outcome } \\
\text { assessment }\end{array}$ & $\begin{array}{l}\text { Incomplete } \\
\text { outcome data }\end{array}$ & $\begin{array}{l}\text { Selective } \\
\text { reporting }\end{array}$ & $\begin{array}{l}\text { Other } \\
\text { bias }\end{array}$ \\
\hline Schurch & 2005 & Low & Low & Low & Low & Low & Low & Unclear \\
\hline Sahai & 2007 & Low & Low & Low & Low & Unclear & Low & Unclear \\
\hline Brubaker & 2008 & Unclear & Unclear & Low & Unclear & Low & Low & Unclear \\
\hline Cohen & 2009 & Low & Low & Low & Unclear & Low & Low & Unclear \\
\hline Dmochowski & 2010 & Low & Unclear & Low & Low & Low & Low & Unclear \\
\hline Rovner & 2011 & Low & Low & Unclear & Low & Low & Low & Unclear \\
\hline Cruz & 2011 & Low & Low & Low & Low & Low & Low & Unclear \\
\hline Herschorn & 2011 & Low & Low & Low & Low & Low & Low & Unclear \\
\hline Altaweel & 2011 & Low & Low & Low & Low & Low & Low & Unclear \\
\hline Denys & 2012 & Low & Unclear & Low & Low & Low & Low & Unclear \\
\hline Ginsberg & 2012 & Low & Low & Low & Low & Low & Low & Unclear \\
\hline Rovner & 2013 & Low & Low & Low & Low & Low & Low & Unclear \\
\hline Nitti & 2013 & Low & Low & Unclear & Low & Low & Low & Unclear \\
\hline Kennelly & 2013 & Low & Low & Low & Low & Low & Low & Unclear \\
\hline Ginsberg & 2013 & Low & Low & Low & Low & Low & Low & Unclear \\
\hline Chapple & 2013 & Low & Low & Low & Unclear & Low & Low & Unclear \\
\hline Sievert & 2014 & Unclear & Low & Low & Unclear & Low & Low & Unclear \\
\hline Chen & 2014 & Low & Low & Low & Unclear & Low & Low & Unclear \\
\hline Abdelwahab & 2015 & Low & Unclear & Low & Unclear & Low & Low & Unclear \\
\hline
\end{tabular}

At 6 weeks. Four studies (Cruz et al., 2011; Ginsberg et al., 2012; Kennelly et al., 2013; Rovner et al., 2013) investigated these outcomes. Figure 3 demonstrated that the BTX-A 300U (MD = $-11.42,95 \%$ CI: $-13.91,-8.93)$ and BTX-A 200U (MD = -10.72, 95\% CI: $-13.40,-8.04)$ were significantly decreased the episodes of UI compared to placebo, nevertheless, the effect between BTXA $300 \mathrm{U}$ and $200 \mathrm{U}(\mathrm{MD}=-0.38,95 \% \mathrm{CI}:-2.60,1.84)$ showed no significant reduction in episodes of UI (Table 3 ).

At 12 weeks. There was only one study (Cruz et al., 2011) reported this outcome. Figure 4 showed that the effect from different dosages of BTX-A were significantly different compared to placebo (200U: $\mathrm{MD}=-8.50,95 \% \mathrm{CI}:-14.46,-2.54$ and $300 \mathrm{U}$ : $\mathrm{MD}=-7.80,95 \% \mathrm{CI}:-13.73,-1.87)$, furthermore, no significant difference between $300 \mathrm{U}$ and $200 \mathrm{U}$ was observed $(\mathrm{MD}=0.70$, 95\%CI: $-4.73,6.13$ ) (Table 3).

\section{Maximum Detrusor Pressure (MDP) MDP for NDO in Short-Term Observation Period}

At 6 weeks. Five included studies (Schurch et al., 2005; Cruz et al., 2011; Ginsberg et al., 2012; Ginsberg et al., 2013; Rovner et al., 2013) reported this outcome. Compared with placebo, BTX-A at both 300U (MD = -31.31, 95\% CI: $-35.79,-26.84)$ and $200 \mathrm{U}$ ( $\mathrm{MD}=-33.01,95 \% \mathrm{CI}:-37.75,-28.27)$ showed a significant effect in reducing MDP for NDO patients. However, the effect of different dosages from BTX-A was not significant (300U versus 200U: $\mathrm{MD}=1.16,95 \% \mathrm{CI}:-3.29,5.60)$ (Figure 5).

\section{$M D P$ for IOAB in Short-Term Observation Period}

At 6 weeks. There was only one study (Rovner et al., 2011) investigated this outcome. And no significant effect between different dosages (50U, 100U, 150U, 200U and 300U) was observed compared to placebo. In addition, statistic difference was merely obtained in two subgroups (BTX-A $150 \mathrm{U}$ vs. $50 \mathrm{U}$ : $\mathrm{MD}=-8.90,95 \% \mathrm{CI}:-16.98,-0.82$ and BTX-A $200 \mathrm{U}$ vs. $150 \mathrm{U}$ :
$\mathrm{MD}=9.90,95 \% \mathrm{CI}: 1.03,18.77)$, meanwhile, the other remaining results showed no statistical difference (Figure 6).

MDP for IOAB in Long-Term Observation Period

At 36 weeks. Only one study (Rovner et al., 2011) reported the outcome. No significant difference was observed in neither different dosages at BTX-A (50U, 100U, 150U, 200U, and $300 \mathrm{U}$ ) nor compared to placebo in IOAB patients (Figure 7).

\section{PVR for NDO in Short-Term Observation Period}

At 2 weeks. Two included studies (Cruz et al., 2011; Rovner et al., 2013) investigated the outcomes. Table 3 showed that BTX-A (200U: $\mathrm{MD}=93.87,95 \% \mathrm{CI}: 63.91,123.84$; and 300U: $\mathrm{MD}=$ 178.22, 95\% CI: $138.50,217.95)$ significantly increased PVR compared to placebo. Furthermore, significant difference was also observed in BTX-A 300U versus $200 \mathrm{U}(\mathrm{MD}=84.13$, 95\% CI: 35.74, 132.51) (Table 3).

\section{Detrusor Compliance (DC)}

\section{DC for NDO in Short-Term Observation Period}

At 6 weeks. Two included studies (Cruz et al., 2011; Rovner et al., 2013) reported this outcomes. Table 3 showed that BTX-A groups (200U and 300U) significantly increased DC compared to placebo (200U: $\mathrm{MD}=66.29,95 \% \mathrm{CI}: 44.24,88.34 ; 300 \mathrm{U}$ : $\mathrm{MD}=56.51,95 \% \mathrm{CI}: 35.00,78.03)$. However, no significant difference was obtained in different dosages of BTX-A (300U vs. 200U: $\mathrm{MD}=-9.79,95 \% \mathrm{CI}:-35.00,15.42)$ (Table 3).

\section{DC for IOAB in Short-Term Observation Period}

At 12 weeks. There was only one study (Rovner et al., 2011) reported this outcome. All of the subgroups showed no significant difference (BTX-A 50U vs. Placebo, $\mathrm{MD}=65.50$, 95\% CI: 23.24, 107.76; $100 \mathrm{U}$ vs. Placebo, $\mathrm{MD}=85.80,95 \% \mathrm{CI}$ : 41.31, 130.29; $200 \mathrm{U}$ vs. Placebo, MD:104.50, 95\% CI: 47.52, 161.48; $300 \mathrm{U}$ vs. Placebo, $\mathrm{MD}=75.80,95 \% \mathrm{CI}: 23.42,128.18$; 
TABLE 3 | Summary of results with different dosage at different observation points for NDO.

\begin{tabular}{|c|c|c|c|c|c|}
\hline Outcomes & Number of RCTs & MD/RR & $95 \% \mathrm{Cl}$ & $I^{2}(\%)$ & $\mathrm{P}$ for $\mathrm{I}^{2}$ \\
\hline \multicolumn{6}{|c|}{ Episodes of Urinary Incontinence (UI) per Week } \\
\hline \multicolumn{6}{|c|}{2 weeks } \\
\hline BTX-A $200 U$ VS. Placebo & 1 & -9.1 & $-14.10,-4.10$ & NA & NA \\
\hline BTX-A $300 U$ VS. Placebo & 1 & -6.1 & $-12.54,0.34$ & NA & NA \\
\hline BTX-A $300 U$ VS. $200 U$ & 1 & 3 & $-3.30,9.30$ & NA & NA \\
\hline \multicolumn{6}{|l|}{6 weeks } \\
\hline BTX-A $200 U$ VS. Placebo & 3 & -10.72 & $-13.40,-8.04$ & 0 & 0.626 \\
\hline BTX-A $300 U$ VS. Placebo & 3 & -11.42 & $-13.91,-8.93$ & 50 & 0.135 \\
\hline BTX-A $300 U$ VS. $200 U$ & 4 & -0.38 & $-2.60,1.84$ & 0 & 0.765 \\
\hline \multicolumn{6}{|l|}{12 weeks } \\
\hline BTX-A $200 U$ VS. Placebo & 1 & -8.5 & $-14.46,-2.54$ & NA & NA \\
\hline BTX-A $300 U$ VS. Placebo & 1 & -7.8 & $-13.73,-1.87$ & NA & NA \\
\hline BTX-A $300 U$ VS. $200 U$ & 1 & 0.7 & $-4.73,6.13$ & NA & NA \\
\hline \multicolumn{6}{|c|}{ Maximum Detrusor Pressure (MDP) } \\
\hline \multicolumn{6}{|c|}{6 weeks } \\
\hline BTX-A $200 U$ VS. Placebo & 5 & -33.01 & $-37.75,-28.27$ & 0 & 0.998 \\
\hline BTX-A $300 U$ VS. Placebo & 5 & -31.31 & $-35.79,-26.84$ & 0 & 0.679 \\
\hline BTX-A $300 U$ VS. $200 U$ & 6 & 1.16 & $-3.29,5.60$ & 0 & 0.831 \\
\hline \multicolumn{6}{|l|}{ Detrusor compliance (DC) } \\
\hline \multicolumn{6}{|l|}{6 weeks } \\
\hline BTX-A $200 U$ VS. Placebo & 2 & 66.29 & $44.24,88.34$ & 0 & 0.837 \\
\hline BTX-A $300 U$ VS. Placebo & 2 & 56.51 & $35.00,78.03$ & 0 & 0.910 \\
\hline BTX-A $300 U$ VS. $200 U$ & 2 & -9.79 & $-35.00,15.42$ & 0 & 0.965 \\
\hline \multicolumn{6}{|l|}{ Post void residual (PVR) } \\
\hline \multicolumn{6}{|l|}{2 weeks } \\
\hline BTX-A $200 U$ VS. Placebo & 2 & 93.87 & $63.91,123.84$ & 0 & 0.887 \\
\hline BTX-A $300 U$ VS. Placebo & 2 & 178.22 & $138.50,217.95$ & 0 & 0.751 \\
\hline BTX-A $300 U$ VS. $200 U$ & 2 & 84.13 & $35.74,132.51$ & 0 & 0.727 \\
\hline \multicolumn{6}{|l|}{ Adverse Events* } \\
\hline \multicolumn{6}{|c|}{ Urinary tract infections (UTI) } \\
\hline BTX-A $200 U$ VS. Placebo & 5 & 1.44 & $1.27,1.62$ & 0 & 0.974 \\
\hline BTX-A $300 U$ VS. Placebo & 6 & 1.51 & $1.35,1.70$ & 0 & 0.720 \\
\hline BTX-A $300 U$ VS. $200 U$ & 6 & 1.07 & $0.97,1.18$ & 0 & 0.909 \\
\hline \multicolumn{6}{|l|}{ Urinary retention } \\
\hline BTX-A $200 U$ VS. Placebo & 4 & 5.85 & $3.84,8.91$ & 0 & 1.000 \\
\hline BTX-A $300 U$ VS. Placebo & 4 & 6.78 & $4.46,10.30$ & 0 & 0.872 \\
\hline BTX-A $300 U$ VS. $200 U$ & 4 & 1.16 & $0.95,1.43$ & 0 & 0.438 \\
\hline \multicolumn{6}{|l|}{ Muscle weakness } \\
\hline BTX-A $200 U$ VS. Placebo & 3 & 1.53 & $0.76,3.06$ & 69.9 & 0.036 \\
\hline BTX-A $300 U$ VS. Placebo & 4 & 3.01 & $1.50,6.02$ & 0 & 0.910 \\
\hline BTX-A $300 U$ VS. $200 U$ & 3 & 1.75 & $1.03,2.97$ & 77.9 & 0.011 \\
\hline \multicolumn{6}{|l|}{ Hematuria } \\
\hline BTX-A $200 U$ VS. Placebo & 5 & 1.59 & $0.97,2.62$ & 0 & 0.980 \\
\hline BTX-A $300 U$ VS. Placebo & 6 & 1.97 & $1.24,3.13$ & 0 & 0.984 \\
\hline BTX-A $300 U$ VS. $200 U$ & 6 & 1.22 & $0.83,1.80$ & 0 & 0.902 \\
\hline
\end{tabular}

NA, Not available.

$200 \mathrm{U}$ vs. $150 \mathrm{U}, \mathrm{MD}=68.10,95 \% \mathrm{CI}: 5.24,130.96 ; 150 \mathrm{U}$ vs. Placebo, MD:36.40, 95\% CI: -8.85, 81.65) (Table 4).

\section{$D C$ for IOAB in Long-Term Observation Period}

At 36 weeks. There was only one included study (Rovner et al., 2011) reported this outcome. Statistically significant difference was observed in three subgroups (BTX-A 50U vs. Placebo, MD = 60.30, 95\% CI: 16.08, 104.52; BTX-A 200U vs. Placebo, $\mathrm{MD}=$ 64.80, 95\% CI: 4.24, 125.36; BTX-A 300U vs. Placebo, $\mathrm{MD}=$ 60.80, 95\% CI: 11.64, 109.96). However, no significant difference was observed in other subgroups (Table 4).

\section{Safety}

All of the effects on adverse events in NDO and IOAB patients were shown in Table 3 and Table 4, respectively.

\section{Urinary Tract Infections (UTI) \\ UTI for NDO}

Seven included studies (Schurch et al., 2005; Cruz et al., 2011; Herschorn et al., 2011; Ginsberg et al., 2012; Ginsberg et al., 2013; Rovner et al., 2013; Chen \& Kuo, 2014) reported the UTI events in NDO patients. And significant effect on increasing UTI was observed in BTX-A 200U (RR $=1.44,95 \% \mathrm{CI}: 1.27,1.62)$ and $300 \mathrm{U}(\mathrm{RR}=1.51,95 \% \mathrm{CI}: 1.35,1.70)$, compared to placebo. Nevertheless, no significant effect was obtained between BTX-A $300 \mathrm{U}$ and $200 \mathrm{U}$ (RR = 1.07, 95\% CI: 0.97, 1.18) (Table 3).

\section{$U T I$ for IOAB}

Eight included studies (Brubaker et al., 2008; Dmochowski et al., 2010; Altaweel et al., 2011; Denys et al., 2012; Chapple et al., 2013; Nitti et al., 2013; Sievert et al., 2014; Abdelwahab et al., 
TABLE 4 | Summary of results with different dosage at different observation points for IOAB.

\begin{tabular}{|c|c|c|c|c|c|}
\hline Outcomes & Number of RCTs & MD/RR & $95 \% \mathrm{Cl}$ & $I^{2}(\%)$ & $\mathrm{P}$ for $\mathrm{I}^{2}$ \\
\hline \multicolumn{6}{|c|}{ Maximum Detrusor Pressure (MDP) } \\
\hline \multicolumn{6}{|c|}{12 weeks } \\
\hline BTX-A 50U VS. Placebo & 1 & 4.7 & $-3.49,12.89$ & NA & NA \\
\hline BTX-A $100 U$ VS. Placebo & 1 & 0.2 & $-7.55,7.95$ & NA & NA \\
\hline BTX-A $150 U$ VS. Placebo & 1 & -4.2 & $-12.70,4.30$ & NA & NA \\
\hline BTX-A $200 U$ VS. Placebo & 1 & 5.7 & $-3.28,14.68$ & NA & NA \\
\hline BTX-A $300 U$ VS. Placebo & 1 & 0.1 & $-9.86,10.06$ & NA & NA \\
\hline BTX-A $100 U$ VS. $50 U$ & 1 & -4.5 & $-11.78,2.78$ & NA & NA \\
\hline BTX-A $150 U$ VS. $50 U$ & 1 & -8.9 & $-16.98,-0.82$ & NA & NA \\
\hline BTX-A $150 U$ VS. $100 U$ & 1 & -4.4 & $-12.03,3.23$ & NA & NA \\
\hline BTX-A $200 U$ VS. $50 U$ & 1 & 1 & $-7.57,9.57$ & NA & NA \\
\hline BTX-A $200 U$ VS. $100 U$ & 1 & 5.5 & $-2.66,13.66$ & NA & NA \\
\hline BTX-A 200U VS. $150 U$ & 1 & 9.9 & $1.03,18.77$ & NA & NA \\
\hline BTX-A 300U VS. $50 U$ & 1 & -4.6 & $-14.20,5.00$ & NA & NA \\
\hline BTX-A 300U VS. $100 U$ & 1 & -0.1 & $-9.33,9.13$ & NA & NA \\
\hline BTX-A 300U VS. $150 U$ & 1 & 4.3 & $-5.57,14.17$ & NA & NA \\
\hline BTX-A $300 U$ VS. $200 U$ & 1 & -5.6 & $-15.88,4.68$ & NA & NA \\
\hline \multicolumn{6}{|l|}{36 weeks } \\
\hline BTX-A 50U VS. Placebo & 1 & 2.6 & $-8.24,13.44$ & NA & NA \\
\hline BTX-A $100 U$ VS. Placebo & 1 & 8 & $-2.52,18.52$ & NA & NA \\
\hline BTX-A 150 U VS. Placebo & 1 & 3.4 & $-7.14,13.94$ & NA & NA \\
\hline BTX-A $200 U$ VS. Placebo & 1 & 3.8 & $-6.66,14.26$ & NA & NA \\
\hline BTX-A $300 U$ VS. Placebo & 1 & 8.1 & $-3.15,19.35$ & NA & NA \\
\hline BTX-A $100 U$ VS. $50 U$ & 1 & 5.4 & $-1.62,12.42$ & NA & NA \\
\hline BTX-A $150 U$ VS. $50 U$ & 1 & 0.8 & $-6.25,7.85$ & NA & NA \\
\hline BTX-A $150 U$ VS. $100 U$ & 1 & -4.6 & $-11.15,1.95$ & NA & NA \\
\hline BTX-A $200 U$ VS. $50 U$ & 1 & 1.2 & $-5.73,8.13$ & NA & NA \\
\hline BTX-A $200 U$ VS. $100 U$ & 1 & -4.2 & $-10.62,2.22$ & NA & NA \\
\hline BTX-A 200U VS. $150 U$ & 1 & 0.4 & $-6.05,6.85$ & NA & NA \\
\hline BTX-A 300U VS. $50 U$ & 1 & 5.5 & $-2.58,13.58$ & NA & NA \\
\hline BTX-A $300 U$ VS. $100 U$ & 1 & 0.1 & $-7.55,7.75$ & NA & NA \\
\hline BTX-A 300U VS. $150 U$ & 1 & 4.7 & $-2.97,12.37$ & NA & NA \\
\hline BTX-A $300 U$ VS. $200 U$ & 1 & 4.3 & $-3.26,11.86$ & NA & NA \\
\hline \multicolumn{6}{|l|}{ Detrusor compliance (DC) } \\
\hline \multicolumn{6}{|l|}{12 weeks } \\
\hline BTX-A 50U VS. Placebo & 1 & 65.5 & $23.24,107.76$ & NA & NA \\
\hline BTX-A $100 U$ VS. Placebo & 1 & 85.8 & $41.31,130.29$ & NA & NA \\
\hline BTX-A $150 U$ VS. Placebo & 1 & 36.4 & $-8.85,81.65$ & NA & NA \\
\hline BTX-A $200 U$ VS. Placebo & 1 & 104.5 & $47.52,161.48$ & NA & NA \\
\hline BTX-A $300 U$ VS. Placebo & 1 & 75.8 & $23.42,128.18$ & NA & NA \\
\hline BTX-A $100 U$ VS. $50 U$ & 1 & 20.3 & $-28.92,69.52$ & NA & NA \\
\hline \multicolumn{6}{|c|}{ Maximum Detrusor Pressure (MDP) } \\
\hline BTX-A $150 U$ VS. $100 U$ & 1 & -49.4 & $-101.21,2.41$ & NA & NA \\
\hline BTX-A $200 U$ VS. $50 U$ & 1 & 39 & $-21.74,99.74$ & NA & NA \\
\hline BTX-A $200 U$ VS. $100 U$ & 1 & 18.7 & $-43.62,81.02$ & NA & NA \\
\hline BTX-A 200U VS. $150 U$ & 1 & 68.1 & $5.24,130.96$ & NA & NA \\
\hline BTX-A $300 U$ VS. $50 U$ & 1 & 10.3 & $-46.15,66.75$ & NA & NA \\
\hline BTX-A 300U VS. $100 U$ & 1 & -10 & $-68.14,48.14$ & NA & NA \\
\hline BTX-A $300 U$ VS. $150 U$ & 1 & 39.4 & $-19.32,98.12$ & NA & NA \\
\hline BTX-A 300U VS. $200 U$ & 1 & -28.7 & $-96.87,39.47$ & NA & NA \\
\hline \multicolumn{6}{|l|}{36 weeks } \\
\hline BTX-A 50U VS. Placebo & 1 & 60.3 & $16.08,104.52$ & NA & NA \\
\hline BTX-A $100 U$ VS. Placebo & 1 & 39.3 & $-6.18,84.78$ & NA & NA \\
\hline BTX-A $150 U$ VS. Placebo & 1 & 24.8 & $-30.63,80.23$ & NA & NA \\
\hline BTX-A $200 U$ VS. Placebo & 1 & 64.8 & $4.24,125.36$ & NA & NA \\
\hline BTX-A $300 U$ VS. Placebo & 1 & 60.8 & $11.64,109.96$ & NA & NA \\
\hline BTX-A $100 U$ VS. $50 U$ & 1 & -21 & $-62.98,20.98$ & NA & NA \\
\hline BTX-A 150U VS. $50 U$ & 1 & -35.5 & $-88.10,17.10$ & NA & NA \\
\hline BTX-A $150 U$ VS. $100 U$ & 1 & -14.5 & $-68.16,39.16$ & NA & NA \\
\hline BTX-A $200 U$ VS. $50 U$ & 1 & 4.5 & $-53.48,62.48$ & NA & NA \\
\hline BTX-A 200U VS. $100 U$ & 1 & 25.5 & $-33.45,84.45$ & NA & NA \\
\hline BTX-A $200 U$ VS. $150 U$ & 1 & 40 & $-26.93,106.93$ & NA & NA \\
\hline BTX-A 300U VS. $50 U$ & 1 & 0.5 & $-45.43,46.43$ & NA & NA \\
\hline
\end{tabular}


TABLE 4 | Continued

\begin{tabular}{|c|c|c|c|c|c|}
\hline Outcomes & Number of RCTs & MD/RR & $95 \% \mathrm{Cl}$ & $I^{2}(\%)$ & $P$ for $I^{2}$ \\
\hline BTX-A 300U VS. $100 U$ & 1 & 21.5 & $-25.65,68.65$ & NA & NA \\
\hline BTX-A 300U VS. $150 U$ & 1 & 36 & $-20.81,92.81$ & NA & NA \\
\hline BTX-A $300 U$ VS. $200 U$ & 1 & -4 & $-65.83,57.83$ & NA & NA \\
\hline \multicolumn{6}{|c|}{ Adverse Events* } \\
\hline \multicolumn{6}{|c|}{ Urinary tract infections (UTI) } \\
\hline BTX-A 50U VS. Placebo & 2 & 1.95 & $0.96,3.98$ & 0 & 0.675 \\
\hline BTX-A $100 U$ VS. Placebo & 5 & 2.55 & $2.09,3.12$ & 0 & 0.656 \\
\hline BTX-A $150 U$ VS. Placebo & 2 & 2.36 & $1.19,4.68$ & 0 & 0.355 \\
\hline BTX-A $200 U$ VS. Placebo & 2 & 2.68 & $1.46,4.93$ & 0 & 0.634 \\
\hline BTX-A $300 U$ VS. Placebo & 1 & 2.12 & $0.98,4.58$ & NA & NA \\
\hline BTX-A $100 U$ VS. $50 U$ & 2 & 0.97 & $0.59,1.59$ & 18.5 & 0.268 \\
\hline BTX-A 150U VS. $50 U$ & 2 & 1.24 & $0.78,1.98$ & 0 & 0.594 \\
\hline BTX-A $150 U$ VS. $100 U$ & 2 & 1.29 & $0.81,2.05$ & 0 & 0.443 \\
\hline BTX-A $200 U$ VS. $50 U$ & 1 & 1.42 & $0.89,2.25$ & NA & NA \\
\hline BTX-A $200 U$ VS. $100 U$ & 3 & 1.44 & $0.94,2.20$ & 0 & 0.685 \\
\hline BTX-A $200 U$ VS. $150 U$ & 1 & 1.09 & $0.72,1.67$ & NA & NA \\
\hline BTX-A 300U VS. $50 U$ & 1 & 1.02 & $0.61,1.71$ & NA & NA \\
\hline BTX-A 300U VS. $100 U$ & 1 & 0.95 & $0.57,1.57$ & NA & NA \\
\hline BTX-A 300U VS. $150 U$ & 1 & 0.79 & $0.49,1.27$ & NA & NA \\
\hline BTX-A $300 U$ VS. $200 U$ & 1 & 0.72 & $0.45,1.14$ & NA & NA \\
\hline \multicolumn{6}{|l|}{ Urinary retention } \\
\hline BTX-A 50U VS. Placebo & 1 & 3.84 & $0.47,31.67$ & NA & NA \\
\hline BTX-A $100 U$ VS. Placebo & 4 & 13.99 & $5.71,34.30$ & 0 & 0.946 \\
\hline BTX-A $150 U$ VS. Placebo & 1 & 12.04 & $1.65,87.85$ & NA & NA \\
\hline BTX-A $200 U$ VS. Placebo & 1 & 9.92 & $1.34,73.29$ & NA & NA \\
\hline BTX-A 300 U VS. Placebo & 1 & 10.95 & $1.50,80.00$ & NA & NA \\
\hline BTX-A $100 U$ VS. $50 U$ & 1 & 2.04 & $0.74,5.57$ & NA & NA \\
\hline BTX-A $150 U$ VS. $50 U$ & 1 & 3.14 & $1.22,8.09$ & NA & NA \\
\hline BTX-A 150U VS. $100 U$ & 1 & 1.54 & $0.75,3.15$ & NA & NA \\
\hline BTX-A $200 U$ VS. $50 U$ & 1 & 2.58 & $0.98,6.84$ & NA & NA \\
\hline BTX-A 200U VS. $100 U$ & 2 & 1.34 & $0.66,2.72$ & 0 & 0.707 \\
\hline BTX-A 200U VS. $150 U$ & 1 & 0.82 & $0.42,1.60$ & NA & NA \\
\hline BTX-A 300U VS. $50 U$ & 1 & 2.85 & $1.10,7.38$ & NA & NA \\
\hline BTX-A 300U VS. $100 U$ & 1 & 1.4 & $0.68,2.88$ & NA & NA \\
\hline BTX-A 300U VS. $150 U$ & 1 & 0.91 & $0.48,1.71$ & NA & NA \\
\hline BTX-A $300 U$ VS. $200 U$ & 1 & 1.1 & $0.56,2.16$ & NA & NA \\
\hline \multicolumn{6}{|c|}{ PVR-related catheterization } \\
\hline BTX-A 50U VS. Placebo & 2 & 1.06 & $0.26,4.27$ & 56.5 & 0.129 \\
\hline BTX-A $100 U$ VS. Placebo & 2 & 2.31 & $0.72,7.37$ & 58.2 & 0.122 \\
\hline BTX-A $150 U$ VS. Placebo & 2 & 2.4 & $0.79,7.30$ & 83.8 & 0.013 \\
\hline BTX-A $200 U$ VS. Placebo & 3 & 15.74 & $3.13,79.31$ & 0 & 0.985 \\
\hline BTX-A $300 U$ VS. Placebo & 1 & 14.93 & $0.89,249.52$ & NA & NA \\
\hline BTX-A $100 U$ VS. $50 U$ & 2 & 2.28 & $0.73,7.10$ & 0 & 0.767 \\
\hline BTX-A $150 U$ VS. $50 U$ & 2 & 2.89 & $0.98,8.48$ & 7.5 & 0.298 \\
\hline BTX-A 150U VS. $100 U$ & 3 & 1.22 & $0.57,2.63$ & 25.7 & 0.26 \\
\hline BTX-A $200 U$ VS. $50 U$ & 1 & 3.95 & $1.17,13.37$ & NA & NA \\
\hline BTX-A 200U VS. $100 U$ & 1 & 1.94 & $0.77,4.86$ & NA & NA \\
\hline BTX-A $200 U$ VS. $150 U$ & 1 & 1.06 & $0.49,2.77$ & NA & NA \\
\hline BTX-A 300U VS. $50 U$ & 1 & 3.05 & $0.87,10.69$ & NA & NA \\
\hline BTX-A $300 U$ VS. $100 U$ & 1 & 1.5 & $0.57,3.93$ & NA & NA \\
\hline BTX-A 300U VS. $150 U$ & 1 & 0.82 & $0.36,1.85$ & NA & NA \\
\hline BTX-A $300 U$ VS. $200 U$ & 1 & 0.77 & $0.35,1.71$ & NA & NA \\
\hline \multicolumn{6}{|l|}{ Hematuria } \\
\hline BTX-A $100 U$ VS. Placebo & 2 & 1.38 & $0.78,2.42$ & 73.7 & 0.051 \\
\hline
\end{tabular}

NA, Not available.

2015) reported this outcome. Four of 15 subgroups showed a significant effect on increasing UTI, compared to placebo (BTXA 100U: $\mathrm{RR}=2.55,95 \%$ CI: 2.09 , 3.12; 150U: $\mathrm{RR}=2.36,95 \% \mathrm{CI}$ : $1.19,4.68$; 200U: $\mathrm{RR}=2.68,95 \%$ CI: $1.46,4.93 ; 300 \mathrm{U}: \mathrm{RR}=2.12$, $95 \%$ CI: $0.98,4.58)$. And no significant difference was observed in the remain 11 subgroups (Table 4).

\section{Urinary Retention \\ Urinary Retention of NDO}

Four included studies (Cruz et al., 2011; Ginsberg et al., 2012; Ginsberg et al., 2013; Rovner et al., 2013) reported available data of this outcome. Significant effect of BTX-A at 200U and 300U was observed compared to placebo $(\mathrm{RR}=5.85,95 \% \mathrm{CI}$ : 3.84 , 


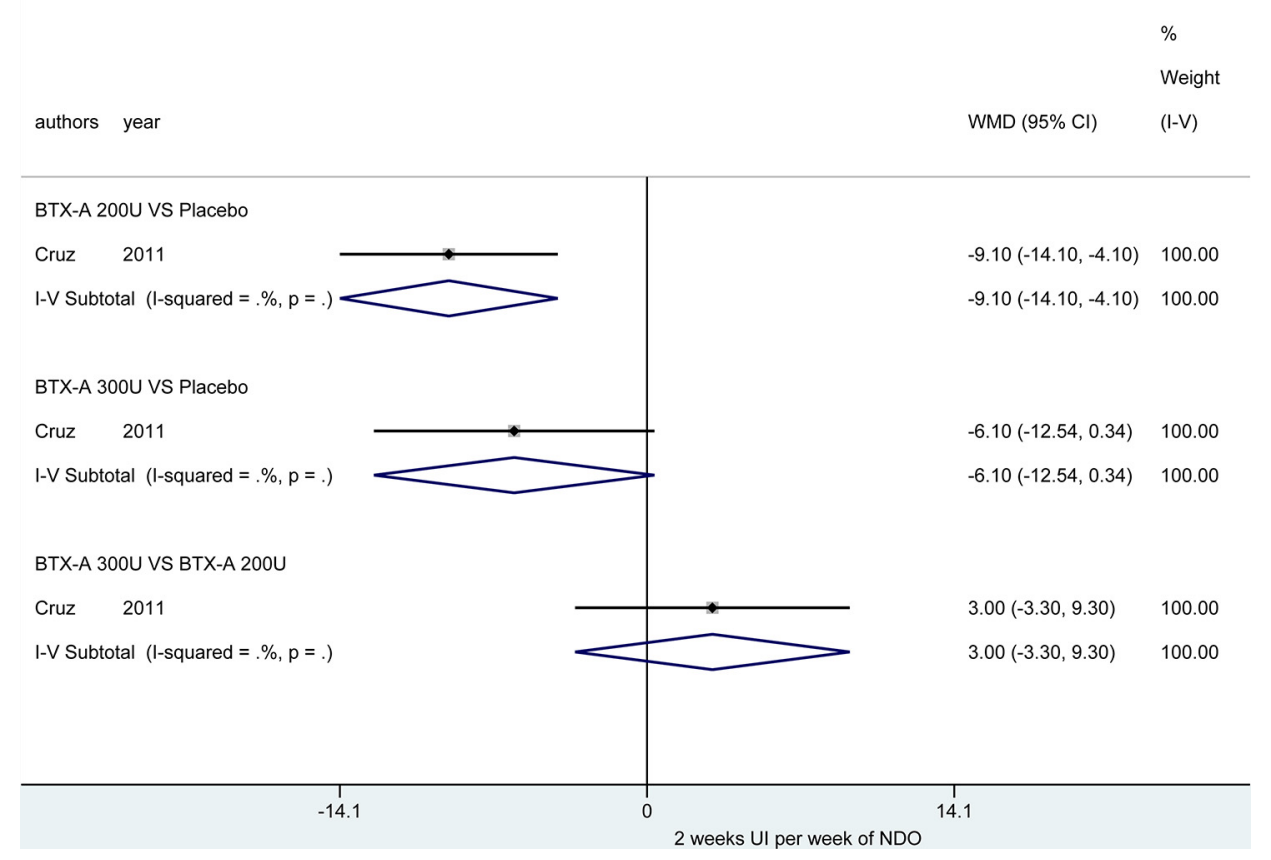

FIGURE 2 | Forest plot of the changes of UI episodes per week of NDO at 2 weeks.

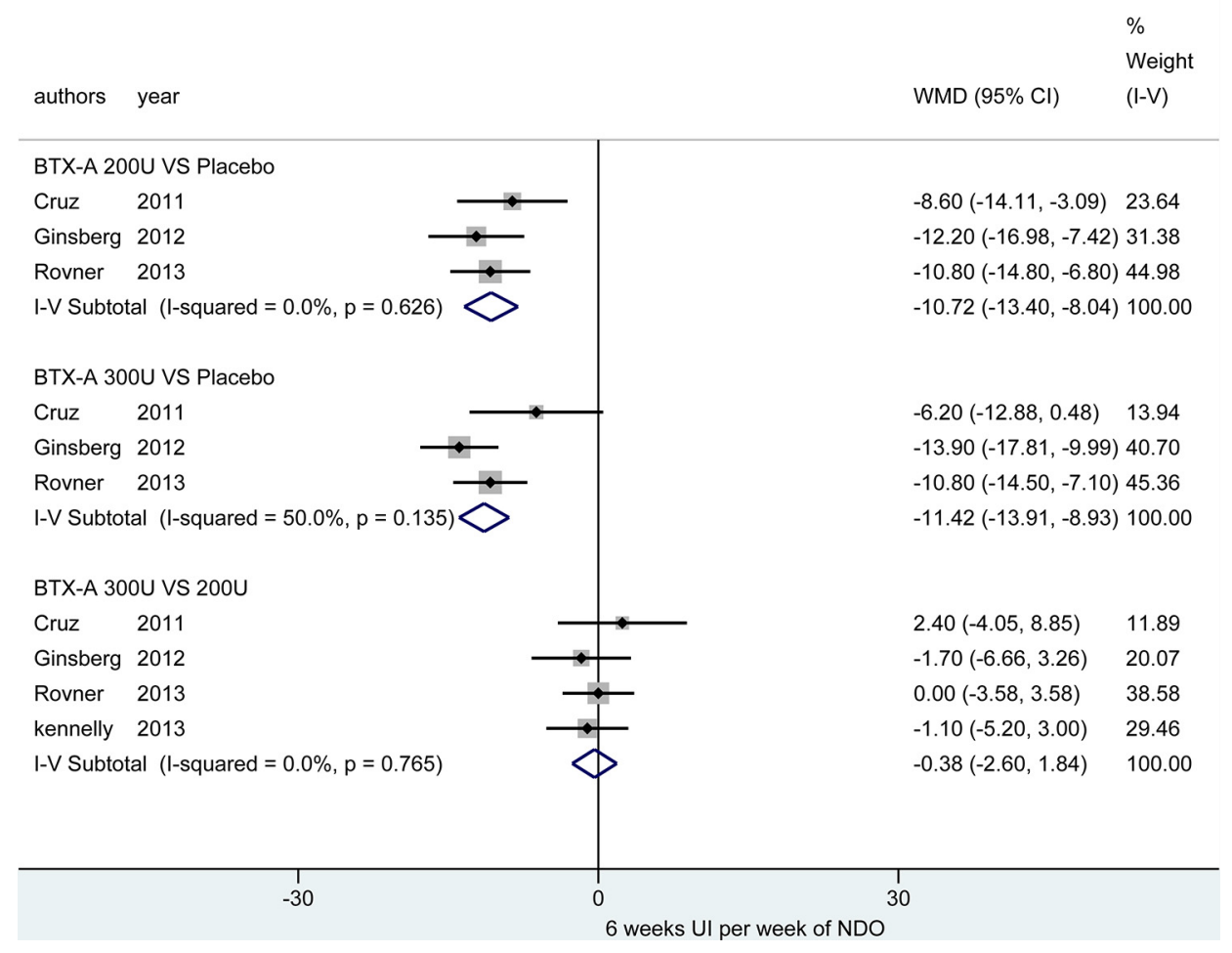

FIGURE 3 | Forest plot of the changes of UI episodes per week of NDO at 6 weeks. 


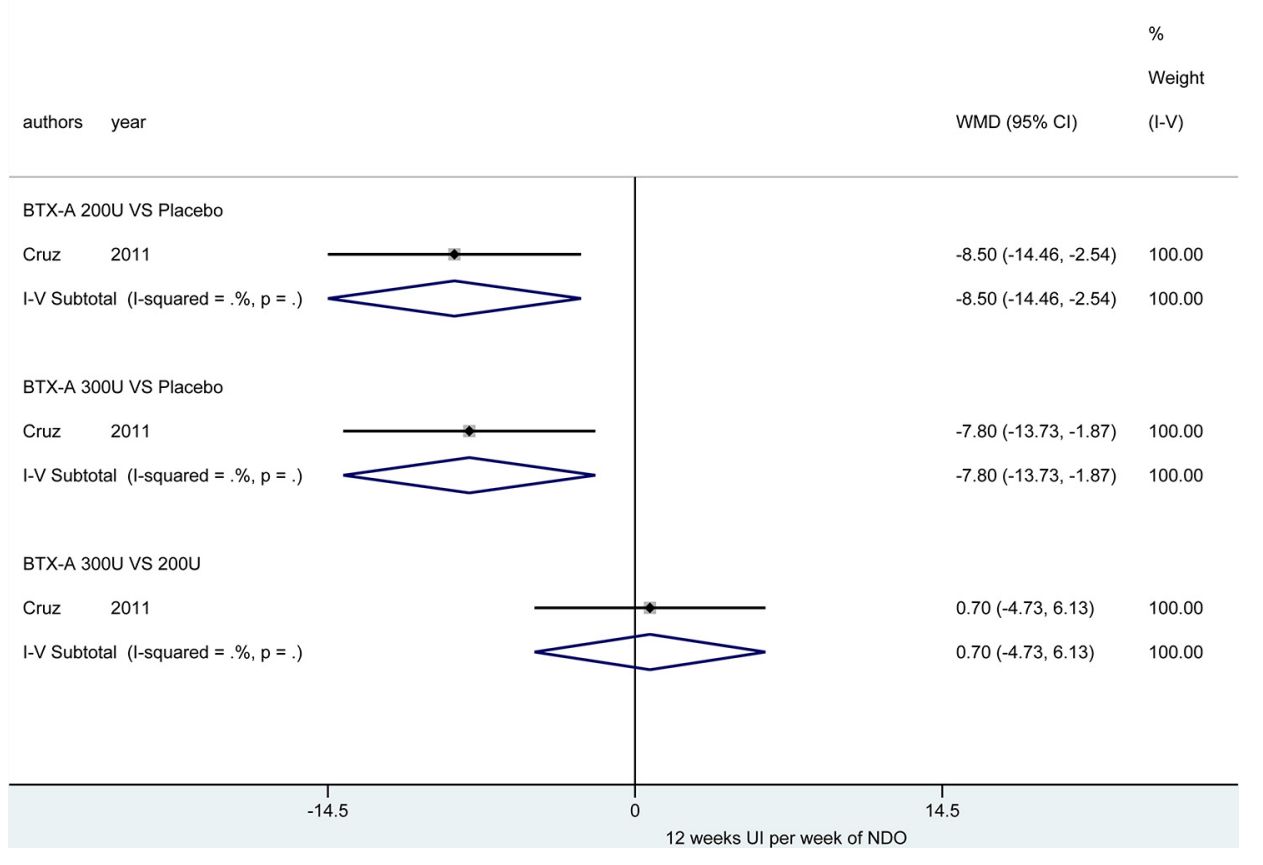

FIGURE 4 | Forest plot of the changes of UI episodes per week of NDO at 12 weeks.

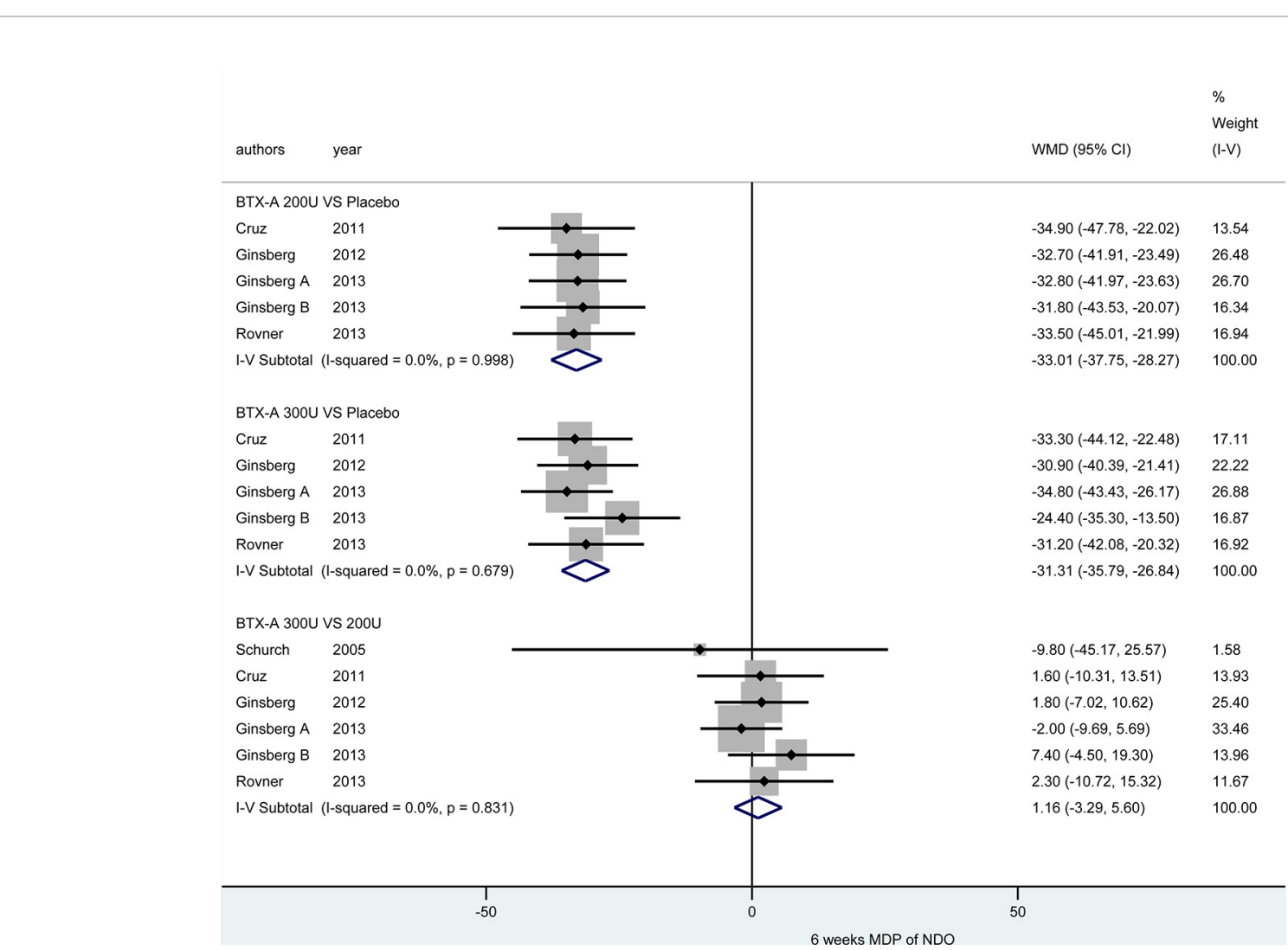

FIGURE 5 | Forest plot of the changes of MDP of NDO at 6 weeks. 


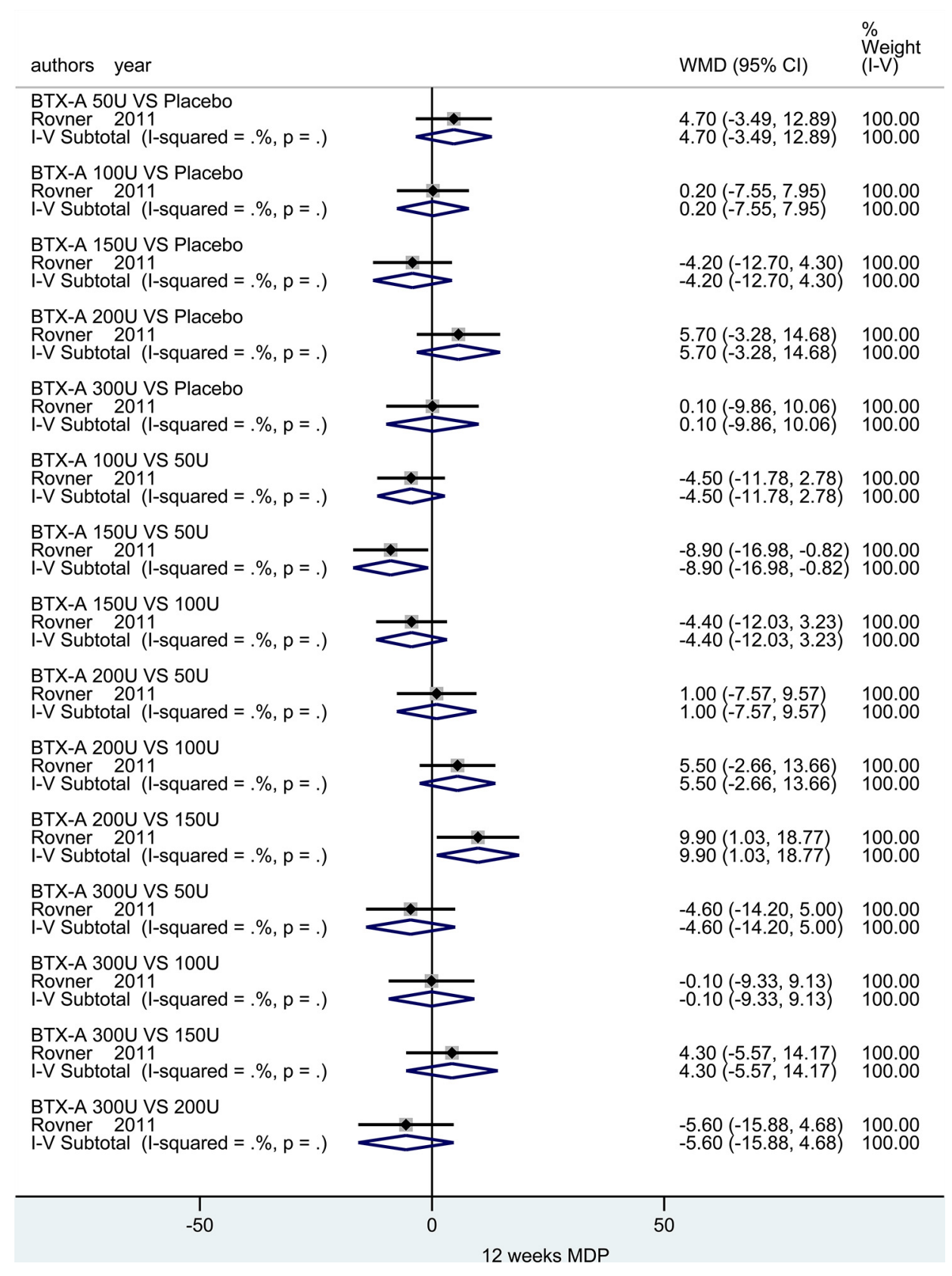

FIGURE 6 | Forest plot of the changes of MDP of IOAB at 12 weeks.

8.91; $\mathrm{RR}=6.78,95 \% \mathrm{CI}: 4.46,10.30)$. Nevertheless, the effect between BTX-A 300U and 200U didn't reach significance $(\mathrm{RR}=$ 1.16, 95\% CI: 0.95, 1.43) (Table 3).

\section{Urinary Retention of $I O A B$}

Four included studies (Dmochowski et al., 2010; Chapple et al., 2013; Nitti et al., 2013; Sievert et al., 2014) reported this outcome. And in 6 of 15 subgroups, BTX-A demonstrated a significant effect on increasing urinary retention (BTX-A 100U vs. Placebo: RR = 13.99, 95\% CI: 5.71, 34.30; 150U vs. Placebo: RR = 12.04, 95\% CI: 1.65, 87.85; 200U vs. Placebo: $R R=9.92,95 \%$ CI: $1.34,73.29 ; 300 U$ vs. Placebo:
$\mathrm{RR}=10.95,95 \%$ CI: $1.50,80.00$; $150 \mathrm{U}$ vs. $50 \mathrm{U}: \mathrm{RR}=3.14,95 \% \mathrm{CI}$ : 1.22, 8.09; 300U vs. 50U: RR = 2.85, 95\% CI: 1.10, 7.38) (Table 4).

\section{Hematuria}

\section{Hematuria of NDO}

Seven included studies (Schurch et al., 2005; Cruz et al., 2011; Herschorn et al., 2011; Ginsberg et al., 2012; Ginsberg et al., 2013; Rovner et al., 2013; Chen \& Kuo, 2014) reported this outcome. The significant difference was only observed in BTX-A 300U, compared to placebo $(\mathrm{RR}=1.97,95 \% \mathrm{CI}: 1.24,3.13)$. For the 


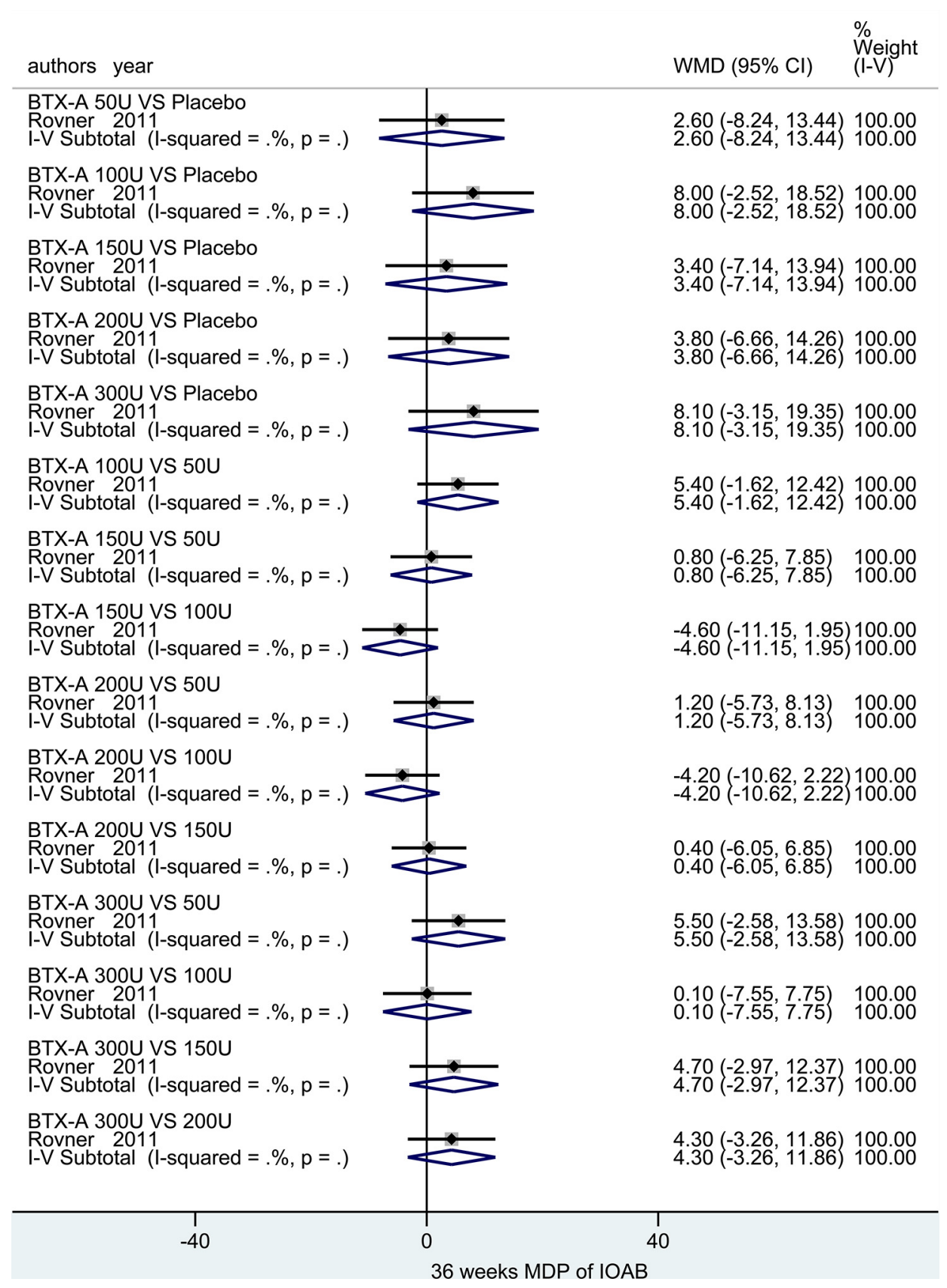

FIGURE 7 | Forest plot of the changes of MDP of IOAB at 36 weeks.

others, no significant difference was observed (BTX-A 200U vs. Placebo: $\mathrm{RR}=1.59,95 \%$ CI: 0.97, 2.62 and $300 \mathrm{U}$ vs. $200 \mathrm{U}: \mathrm{RR}=$ 1.22, 95\% CI: 0.83, 1.80) (Table 3).

\section{Hematuria of $I O A B$}

Two included studies (Chapple et al., 2013; Sievert et al., 2014) reported this outcome. Nevertheless, no significant effect was observed in BTX-A 100U compared to placebo ( $\mathrm{RR}=1.38,95 \%$ CI: $0.78,2.42$ ) (Table 4).

\section{PVR-Related Catheterization of IOAB}

Five included studies (Sahai et al., 2007; Brubaker et al., 2008; Cohen et al., 2009; Dmochowski et al., 2010; Denys et al., 2012; Nitti et al., 2013) reported the available data for PVR-related catheterization in IOAB patients. Significant effect was observed in 2 of 15 subgroups (BTX-A 200U vs. Placebo: RR = 15.74, 95\% CI: 3.13, 79.31; BTX-A 200U vs. 50U: RR = 3.95, 95\% CI: 1.17 , 13.37). And the effect of remain 13 subgroups didn't reach significance (Table 4). 


\section{Muscle Weakness of NDO}

Four included studies (Cruz et al., 2011; Herschorn et al., 2011; Ginsberg et al., 2012; Rovner et al., 2013) reported the available data for muscle weakness in NDO patients. Significant effect on increasing muscle weakness in BTX-A at $300 \mathrm{U}$ was observed compared to placebo ( $\mathrm{RR}=3.01,95 \% \mathrm{CI}: 1.50,6.02)$, however, the effect of BTX-A at $200 \mathrm{U}$ was not significant $(\mathrm{RR}=1.53,95 \% \mathrm{CI}$ : $0.76,3.06)$. Furthermore, the effect of $300 \mathrm{U}$ was statistic significant compared to $200 \mathrm{U}(\mathrm{RR}=1.75,95 \% \mathrm{CI}$ : 1.03, 2.97) (Table 3).

\section{DISCUSSION}

Based on the American Urological Association (AUA) guidelines (Gormley et al., 2015) for management of OAB patients, the standard treatment, including education, behavior therapies, pharmacotherapy, and BTX-A, has been widely used for OAB (Engeler et al., 2015; Zhou et al., 2015; Krhut et al., 2016). We performed this meta-analysis with the most up-to-date and comprehensive evidence for evaluating clinical benefit of BTX-A in UI in patients with NDO and IOAB. In order to reflect the effects more accurately, the different follow-up periods and dosages were analyzed. Ultimately, we found that the BTX-A demonstrated a significant effect on reducing episodes of UI and MDP in NDO patients, and BTX-A increased DC in IOAB patients, however, the adverse events in two groups increased in this meta-analysis.

Five kinds of dosages of BTX-A for NDO and IOAB (50U, $100 \mathrm{U}, 150 \mathrm{U}, 200 \mathrm{U}$, and 300U) and placebo form 19 included studies considered as high quality under quality assessment were analyzed for evaluating the effectiveness. The results demonstrated that BTX-A $300 \mathrm{U}$ and $200 \mathrm{U}$ is superior to placebo in the protective role against UI episodes per week in NDO patients during follow-up period (2, 6, and 12 weeks), which was considered as a satisfactory outcome and enable BTX-A to be considered as intervention for management of UI. Furthermore, BTX-A reduced MDP during 6 weeks follow-up compared to placebo, which might account for the increase in PVR in both $200 \mathrm{U}$ and $300 \mathrm{U}$, and the $300 \mathrm{U}$ was worse than $200 \mathrm{U}$. The DC in NDO was increased at both BTX-A 200U and 300U, which might account for the reduction in episodes of UI. These results were consistent with other similar meta-analyses (Zhou et al., 2015; Cheng et al., 2016). However, the BTX-A $300 \mathrm{U}$ showed no superior effect on UI, MDP, and DC compared to 200U, with increased UTI, urinary retention, muscle weakness, and hematuria, thus, the BTX-A $300 \mathrm{U}$ was not recommended in management of UI in NDO patients in this study.

Furthermore, the results also revealed that dosages of BTX-A not less than $50 \mathrm{U}$ were superior to placebo in the improvement of DC in IOAB at 12 and 36 weeks, except the BTX-A $150 \mathrm{U}$ and $100 \mathrm{U}$ at 36 weeks and BTX-A $150 \mathrm{U}$ at 12 weeks in this study. This superior effect was consistent with the Rovner's study (Rovner et al., 2011) which has reported that BTX-A at dosages of more than $100 \mathrm{U}$ showed significant improvement in $\mathrm{OAB}$. It was also observed between BTX-A 200U and 300U for UI episodes per week at 2 weeks in NDO patients, which may be caused by small sample size. However, compared with placebo, five dosages of BTX-A demonstrated no significant difference in for MDP in IOAB at 12 and 36 weeks, which was not consistent with Chapple's study (Chapple et al., 2013) reported OAB patients could be benefit from BTX-A 100U. In addition, BTXA 200U and 300U had nearly same effectiveness on NDO and IOAB. Although Nuanthaisong's study (Nuanthaisong et al., 2014) reported that BTX-A over $360 \mathrm{U}$ was an effective treatment, we mainly focused on dosages not more than 300 , especially 300 and $200 \mathrm{U}$ for NDO and IOAB, thus, the studies exploring effect of dosages of BTX-A more than $300 \mathrm{U}$ are required in further research. All these results suggested that BTX-A $300 \mathrm{U}$ and $200 \mathrm{U}$ is superior to placebo in effectiveness on NDO at short-term observations, especially at 6 weeks. Remarkably, in consideration that UI was regarded as a chronic disease, and BTX-A 200U also contributed to increase $\mathrm{DC}$ in IOAB patients during 36 weeks, therefore, the researches exploring long-term effect of BTX-A are required in future.

Another essential part of this study was the assessment of safety of BTX-A, and we found that basically all the adverse events were increased in both NDO and IOAB patients in this meta-analysis. The results showed that possibility of suffering UTI was higher in both BTX-A 300U and 200U in NDO than placebo, but the BTXA $300 \mathrm{U}$ was not higher than 200U. This effect might due to the markedly increased PVR and urinary retention. Therefore, the NDO patients treated with BTX-A must be aware of the potential risk of UTI, and the prophylactic antibiotics could be considered. These complications could account for the increased risk of hematuria as consequence, except in BTX-A 200U. And no effect on increasing risk of muscle weakness was observed in BTX-A 200U in NDO patients, however the BTX-A 300U significantly increased this risk compared to both placebo and BTX-A 200U. For IOAB patients, almost every dosages of BTX-A, except at $50 \mathrm{U}$, demonstrated a higher risk of urinary retention compared to placebo. Furthermore, the increased dosages basically did not increase the risk of urinary retention. Therefore, in theory, the different dosages would not increase the risk of UTI as consequence, which was consistent with the results of this study that the risk of suffering UTI was higher than placebo, however, no increased risk was observed in different dosages. In addition, no requirement of PVR-related catheterizations was observed in IOAB patients compared to placebo, except at dosage of $200 \mathrm{U}$. The exception and heterogeneity may be caused by small sample size. Although BTX-A was superior to placebo, indicating that BTX-A had slightly more adverse events than placebo. However, the localized urologic events, which considered as main adverse effects of BTX-A, were not found. It might due to the welltolerated, however, more researches are still required in this field.

The advantages in this study are as follows. Firstly, Chinese articles were also reviewed in this meta-analysis as well as English studies, unfortunately, there were no studies met the inclusion criteria. Secondly, BTX-A at different dosages were investigated, and the clinical benefit of BTX-A was assessed based on comprehensive measurement of outcomes. Thus, the most upto-date evidence has been provided for clinical practice and medical guidelines. Meanwhile, different dosages were evaluated to assess the safety. In our study, the results demonstrated that 
the increase of dosage of BTX-A has no protective effect of complications. For effectiveness, although the BTX-A has a protective effect for NDO, different dosage showed no significant discrepancy in UI episodes, MDP and DC, except PVR. For IOAB, the most remarkable outcome is the difference of PVR for injecting diverse dosage. In order to detail the longterm potential impact, we comprehensively and systematically evaluated BTX-A in treatment of UI in patients with NDO and IOAB for short- and long-term observation periods, which could reduce the risk of from correct outcomes as well. Although significant differences were not obtained in our study, we offered a potential possibility for other researcher to investigate this field for the prognosis of patients. Furthermore, results of subgroup analyses based on dosages and types of OAB objectively disclosure true the clinical benefit, and it has guiding clinicians to treat NDO and IOAB. After all, Surgery may be carried out as a last resort (Krhut et al., 2016), resulting in trauma, thus the drug treatment of $\mathrm{OAB}$ requires urgent attention.

There are also several limitations in this study. Firstly, insufficient sample size and RCTs is the main disadvantages in our study, caused by the study design of original studies. For example, we excluded one study reported results $72 \mathrm{~h}$ after injection, which is possible to ignore the long-term impact which might produce the result of greater difference. Due to the limitations of language, the studies using other languages (except English and Chinese) were ignored. Secondly, benign prostatic hyperplasia $(\mathrm{BPH})$ is a prominent factor, resulting $\mathrm{OAB}$. The pathogenesis was failed to be executed using subgroup analyses, because of the lack of relevant data. The data of short-term and long-term observations periods were insufficient in assess the clinical benefits of BTX-A. Therefore, more high-quality larger RCTs with short-term and long-term observations periods should be added to assess of the clinical benefits of BTX-A from different perspectives.

\section{REFERENCES}

Abdelwahab, O., Sherif, H., Soliman, T., Elbarky, I., and Eshazly, A. (2015). Efficacy of botulinum toxin type A 100 Units versus 200 units for treatment of refractory idiopathic overactive bladder. Int. Braz. J. Urol. 41, 1132-1140. doi: 10.1590/S1677-5538.IBJU.2014.0221

Abrams, P., Cardozo, L., Fall, M., Griffiths, D., Rosier, P., Ulmsten, U., et al. (2002). The standardisation of terminology of lower urinary tract function: report from the standardisation sub-committee of the international continence society. Am. J. Obstet. Gynecol. 187, 116-126. doi: 10.1067/mob.2002.125704

Altaweel, W., Mokhtar, A., and Rabah, D. M. (2011). Prospective randomized trial of $100 \mathrm{u}$ vs $200 \mathrm{u}$ botox in the treatment of idiopathic overactive bladder. Urol. Ann. 3, 66-70. doi: 10.4103/0974-7796.82170

Asimakopoulos, A. D., Cerruto, M. A., Del Popolo, G., La Martina, M., Artibani, W., Carone, R., et al. (2012). An overview on mixed action drugs for the treatment of overactive bladder and detrusor overactivity. Urol. Int. 89, 259269. doi: 10.1159/000339600

Brubaker, L., Richter, H. E., Visco, A., Mahajan, S., Nygaard, I., Braun, T. M., et al. (2008). Refractory idiopathic urge urinary incontinence and botulinum A injection. J. Urol. 180, 217-222. doi: 10.1016/j.juro.2008.03.028

Chapple, C., Sievert, K. D., MacDiarmid, S., Khullar, V., Radziszewski, P., Nardo, C., et al. (2013). OnabotulinumtoxinA $100 \mathrm{U}$ significantly improves all idiopathic overactive bladder symptoms and quality of life in patients with overactive

\section{CONCLUSION}

This meta-analysis indicates that BTX-A 300U and 200U showed a positive effect on management of UI NDO for short-term therapy based on current evidence. In addition, clinical benefits were not found in BTX-A 50U, 100U, 150U, 200U, and 300U for IOAB for long-term treatment, except the efficacy of BTX-A for DC. There is a significant finding that dosages $300 \mathrm{U}$ and $200 \mathrm{U}$ could improve DC of IOAB for short- and long-term treatments, compared to placebo. Furthermore, BTX-A $300 \mathrm{U}$ and $200 \mathrm{U}$ have no significant difference in adverse events. Therefore, we recommend that BTX-A $200 \mathrm{U}$ could be considered as intervention for UI in patients with NDO for the short-term treatments, with minimal, local, and manageable adverse events. The long-term treatments of BTX-A for UI in NDO patients and short-term treatments for IOAB patients require more RCTs to be investigated.

\section{AUTHOR CONTRIBUTIONS}

Q-QG, X-YD, Y-QX and JX had full access to the data and take responsibility for the integrity and accuracy of the data analysis. Q-QG, X-YD and JX were responsible for the study concept and design. CG, Y-QX and JX investigators/collaborators listed below were involved in the acquisition of data. All the authors contributed to the analysis and interpretation of data and to the critical revision of manuscript. CG drafted the manuscript.

\section{SUPPLEMENTARY MATERIALS}

The Supplementary Material for this article can be found online at: https://www.frontiersin.org/articles/10.3389/fphar. 2019.01618/full\#supplementary-material

bladder and urinary incontinence: a randomised, double-blind, placebocontrolled trial. Eur. Urol. 64, 249-256. doi: 10.1016/j.eururo.2013.04.001

Chen, Y. C., and Kuo, H. C. (2014). The therapeutic effects of repeated detrusor injections between 200 or 300 units of onabotulinumtoxinA in chronic spinal cord injured patients. Neurourol. Urodyn. 33, 129-134. doi: 10.1002/nau.22395

Cheng, T., Shuang, W. B., Jia, D. D., Zhang, M., Tong, X. N., Yang, W. D., et al. (2016). Efficacy and safety of OnabotulinumtoxinA in patients with neurogenic detrusor overactivity: a systematic review and meta-analysis of randomized controlled trials. PloS One 11, e0159307. doi: 10.1371/journal.pone.0159307

Cohen, B. L., Barboglio, P., Rodriguez, D., and Gousse, A. E. (2009). Preliminary results of a dose-finding study for botulinum toxin-A in patients with idiopathic overactive bladder: 100 versus 150 units. Neurourol. Urodyn. 28, 205-208. doi: 10.1002/nau.20611

Cruz, F., Herschorn, S., Aliotta, P., Brin, M., Thompson, C., Lam, W., et al. (2011). Efficacy and safety of onabotulinumtoxin $A$ in patients with urinary incontinence due to neurogenic detrusor overactivity: a randomised, double-blind, placebocontrolled trial. Eur. Urol. 60, 742-750. doi: 10.1016/j.eururo.2011.07.002

Cui, Y., Wang, L., Liu, L., Zeng, F., Niu, J., Qi, L., et al. (2013). Botulinum toxin-A injections for idiopathic overactive bladder: a systematic review and metaanalysis. Urol. Int. 91, 429-438. doi: 10.1159/000351037

Cui, Y., Zhou, X., Zong, H., Yan, H., and Zhang, Y. (2015). The efficacy and safety of onabotulinumtoxinA in treating idiopathic OAB: a systematic review and meta-analysis. Neurourol. Urodyn. 34, 413-419. doi: 10.1002/nau.22598 
Deeks, J. (2002). Issues in the selection of a summary statistic for meta-analysis of clinical trials with binary outcomes. Stat. Med. 21, 1575-1600. doi: 10.1002/ $\operatorname{sim} .1188$

Denys, P., Le Normand, L., Ghout, I., Costa, P., Chartier-Kastler, E., Grise, P., et al. (2012). Efficacy and safety of low doses of onabotulinumtoxinA for the treatment of refractory idiopathic overactive bladder: a multicentre, doubleblind, randomised, placebo-controlled dose-ranging study. Eur. Urol. 61, 520529. doi: 10.1016/j.eururo.2011.10.028

Dmochowski, R., Chapple, C., Nitti, V. W., Chancellor, M., Everaert, K., Thompson, C., et al. (2010). Efficacy and safety of onabotulinumtoxinA for idiopathic overactive bladder: a double-blind, placebo controlled, randomized, dose ranging trial. J. Urol. 184, 2416-2422. doi: 10.1016/j.juro.2010.08.021

Durden, E., and Walker, D. (2018). The economic burden of overactive bladder $(\mathrm{OAB})$ and its effects on the costs associated with other chronic, age-related comorbidities in the United States. Neurourol. Urodyn. 37, 1641-1649. doi: 10.1002/nau.23513

Engeler, D. S., Meyer, D., Abt, D., Muller, S., and Schmid, H. P. (2015). Sacral neuromodulation for the treatment of neurogenic lower urinary tract dysfunction caused by multiple sclerosis: a single-centre prospective series. BMC Urol. 15, 105. doi: 10.1186/s12894-015-0102-x

Flynn, M. K., Webster, G. D., and Amundsen, C. L. (2004). The effect of botulinum-A toxin on patients with severe urge urinary incontinence. $J$. Urol. 172, 2316-2320. doi: 10.1097/01.ju.0000143889.00681.3f

Garely, A. D., and Burrows, L. J. (2002). Current pharmacotherapeutic strategies for overactive bladder. Expert Opin. Pharmacother. 3, 827-833. doi: 10.1517/ 14656566.3.7.827

Ginsberg, D., Gousse, A., Keppenne, V., Sievert, K. D., Thompson, C., Lam, W., et al. (2012). Phase 3 efficacy and tolerability study of onabotulinumtoxinA for urinary incontinence from neurogenic detrusor overactivity. J. Urol. 187, 21312139. doi: 10.1016/j.juro.2012.01.125

Ginsberg, D., Cruz, F., Herschorn, S., Gousse, A., Keppenne, V., Aliotta, P., et al. (2013). OnabotulinumtoxinA is effective in patients with urinary incontinence due to neurogenic detrusor overactivity [corrected] regardless of concomitant anticholinergic use or neurologic etiology. Adv. Ther. 30, 819-833. doi: 10.1007/s12325-013-0054-z

Gormley, E. A., Lightner, D. J., Faraday, M., and Vasavada, S. P. (2015). Diagnosis and treatment of overactive bladder (non-neurogenic) in adults: AUA/SUFU guideline amendment. J. Urol. 193, 1572-1580. doi: 10.1016/j.juro.2012.09.079

Gu, H. Y., Song, J. K., Zhang, W. J., Xie, J., Yao, Q. S., Zeng, W. J., et al. (2017). A systematic review and meta-analysis of effectiveness and safety of therapy for overactive bladder using botulinum toxin A at different dosages. Oncotarget. 8, 90338-90350. doi: 10.18632/oncotarget.20056

Herschorn, S., Gajewski, J., Ethans, K., Corcos, J., Carlson, K., Bailly, G., et al. (2011). Efficacy of botulinum toxin A injection for neurogenic detrusor overactivity and urinary incontinence: a randomized, double-blind trial. J. Urol. 185, 2229-2235. doi: 10.1016/j.juro.2011.02.004

Higgins, J. P., Altman, D. G., Gotzsche, P. C., Juni, P., Moher, D., Oxman, A. D., et al. (2011). The Cochrane Collaboration's tool for assessing risk of bias in randomised trials. Bmj 343, d5928. doi: 10.1136/bmj.d5928

Higgins JP, T. S. (2002). Quantifying heterogeneity in a meta-analysis. Stat. Med. 21, 1539-1558. doi: 10.1111/1469-0691.12494

Higgins JP, G. S. (2011). Cochrane handbook for systematic reviews of interventions, v.5.1, (United Kingdom: Cochrane Library). Available from: http://handbook-5-1.cochrane.org/. [Last updated on 2011 Mar 05].

Kennelly, M., Dmochowski, R., Ethans, K., Karsenty, G., Schulte-Baukloh, H., Jenkins, B., et al. (2013). Long-term efficacy and safety of onabotulinumtoxinA in patients with urinary incontinence due to neurogenic detrusor overactivity: an interim analysis. Urology. 81, 491-497. doi: 10.1016/j.urology.2012.11.010

Kessler, T. M., Danuser, H., Schumacher, M., Studer, U. E., and Burkhard, F. C. (2005). Botulinum A toxin injections into the detrusor: an effective treatment in idiopathic and neurogenic detrusor overactivity? Neurourol. Urodyn. 24, 231-236. doi: 10.1002/nau.20105

Krhut, J., Navratilova, M., Sykora, R., Jurakova, M., Gartner, M., Mika, D., et al. (2016). Intravesical instillation of onabotulinum toxin A embedded in inert hydrogel in the treatment of idiopathic overactive bladder: A double-blind randomized pilot study. Scand. J. Urol. 50, 200-205. doi: 10.3109/ 21681805.2015.1121406
Kuo, H. C. (2005). Clinical effects of suburothelial injection of botulinum A toxin on patients with nonneurogenic detrusor overactivity refractory to anticholinergics. Urol. 66, 94-98. doi: 10.1016/j.urology.2005.02.002

Lawrence, J. M., Lukacz, E. S., Nager, C. W., Hsu, J. W., and Luber, K. M. (2008). Prevalence and co-occurrence of pelvic floor disorders in community-dwelling women. Obstet. Gynecol. 111, 678-685. doi: 10.1097/AOG.0b013e3181660c1b

Majumdar, A T-HP (2004). Detrusor overactivity. Curr. Obstet. Gynaecol. 14, 395404.

Mehta, S., Hill, D., McIntyre, A., Foley, N., Hsieh, J., Ethans, K., et al. (2013). Metaanalysis of botulinum toxin A detrusor injections in the treatment of neurogenic detrusor overactivity after spinal cord injury. Arch. Phys. Med. Rehabil. 94, 1473-1481. doi: 10.1016/j.apmr.2013.04.011

Melsen, W. G., Bootsma, M. C., Rovers, M. M., and Bonten, M. J. (2014). The effects of clinical and statistical heterogeneity on the predictive values of results from meta-analyses. Clin. Microbiol. Infect.: Off. Publ. Eur. Soc. Clin. Microbiol. Infect. Dis. 20, 123-129. doi: 10.1111/1469-0691.12494

Moher, D., Liberati, A., Tetzlaff, J., and Altman, D. G. (2009). Preferred reporting items for systematic reviews and meta-analyses: the PRISMA statement. BMJ 339, b2535. doi: 10.1136/bmj.b2535

Nitti, V. W., Dmochowski, R., Herschorn, S., Sand, P., Thompson, C., Nardo, C., et al. (2013). OnabotulinumtoxinA for the treatment of patients with overactive bladder and urinary incontinence: results of a phase 3, randomized, placebo controlled trial. J. Urol. 189, 2186-2193. doi: 10.1016/ j.juro.2012.12.022

Nuanthaisong, U., Abraham, N., and Goldman, H. B. (2014). Incidence of adverse events after high doses of onabotulinumtoxinA for multiple indications. Urol. 84, 1044-1048. doi: 10.1016/j.urology.2014.07.046

Popat, R., Apostolidis, A., Kalsi, V., Gonzales, G., Fowler, C. J., and Dasgupta, P. (2005). A comparison between the response of patients with idiopathic detrusor overactivity and neurogenic detrusor overactivity to the first intradetrusor injection of botulinum-A toxin. J. Urol. 174, 984-989. doi: 10.1097/01.ju.0000169480.43557.31

Rajkumar, G. N., Small, D. R., Mustafa, A. W., and Conn, G. (2005). A prospective study to evaluate the safety, tolerability, efficacy and durability of response of intravesical injection of botulinum toxin type A into detrusor muscle in patients with refractory idiopathic detrusor overactivity. BJU Int. 96, 848852. doi: 10.1111/j.1464-410X.2005.05725.x

Rovner, E., Kennelly, M., Schulte-Baukloh, H., Zhou, J., Haag-Molkenteller, C., and Dasgupta, P. (2011). Urodynamic results and clinical outcomes with intradetrusor injections of onabotulinumtoxinA in a randomized, placebocontrolled dose-finding study in idiopathic overactive bladder. Neurourol. Urodyn. 30, 556-562. doi: 10.1002/nau.21021

Rovner, E., Dmochowski, R., Chapple, C., Thompson, C., Lam, W., and HaagMolkenteller, C. (2013). OnabotulinumtoxinA improves urodynamic outcomes in patients with neurogenic detrusor overactivity. Neurourol. Urodyn. 32, 1109-1115. doi: 10.1002/nau.22376

Sahai, A., Khan, M. S., and Dasgupta, P. (2007). Efficacy of botulinum toxin-A for treating idiopathic detrusor overactivity: results from a single center, randomized, double-blind, placebo controlled trial. J. Urol. 177, 2231-2236. doi: 10.1016/j.juro.2007.01.130

Schmid, D. M., Sauermann, P., Werner, M., Schuessler, B., Blick, N., Muentener, M., et al. (2006). Experience with 100 cases treated with botulinum-A toxin injections in the detrusor muscle for idiopathic overactive bladder syndrome refractory to anticholinergics. J. Urol. 176, 177-185. doi: 10.1016/S0022-5347 (06)00590-8

Schulte-Baukloh, H., Weiss, C., Stolze, T., Herholz, J., Sturzebecher, B., Miller, K., et al. (2005). Botulinum-A toxin detrusor and sphincter injection in treatment of overactive bladder syndrome: objective outcome and patient satisfaction. Eur. Urol. 48, 984-990. discussion 90. doi: 10.1016/j.eururo.2005.06.021

Schurch, B., de Seze, M., Denys, P., Chartier-Kastler, E., Haab, F., Everaert, K., et al. (2005). Botulinum toxin type a is a safe and effective treatment for neurogenic urinary incontinence: results of a single treatment, randomized, placebo controlled 6-month study. J. Urol. 174, 196-200. doi: 10.1097/01.ju.0000162035.73977.1c

Sievert, K. D., Chapple, C., Herschorn, S., Joshi, M., Zhou, J., Nardo, C., et al. (2014). OnabotulinumtoxinA $100 \mathrm{U}$ provides significant improvements in overactive bladder symptoms in patients with urinary incontinence regardless of the number of anticholinergic therapies used or reason for 
inadequate management of overactive bladder. Int. J. Clin. Pract. 68, 12461256. doi: $10.1111 /$ ijcp. 12443

Stewart, W. F., Van Rooyen, J. B., Cundiff, G. W., Abrams, P., Herzog, A. R., Corey, R., et al. (2003). Prevalence and burden of overactive bladder in the United States. World J. Urol. 20, 327-336. doi: 10.1007/s00345-002-0301-4

Sun, Y., Luo, D., Tang, C., Yang, L., and Shen, H. (2015). The safety and efficiency of onabotulinumtoxinA for the treatment of overactive bladder: a systematic review and meta-analysis. Int. Urol. Nephrol. 47, 1779-1788. doi: 10.1007/ s11255-015-1125-7

White, N., and Iglesia, C. B. (2016). Overactive Bladder. Obstet. Gynecol. Clin. North Am. 43, 59-68. doi: 10.1016/j.ogc.2015.10.002

Zhang, R., Xu, Y., Yang, S., Liang, H., Zhang, Y., and Liu, Y. (2015). OnabotulinumtoxinA for neurogenic detrusor overactivity and dose differences: a systematic review. Int. Braz. J. Urol. 41, 207-219. doi: 10.1590/ S1677-5538.IBJU.2015.02.05
Zhou, X., Yan, H. L., Cui, Y. S., Zong, H. T., and Zhang, Y. (2015). Efficacy and safety of onabotulinumtoxinA in treating neurogenic detrusor overactivity: a systematic review and meta-analysis. Chin. Med. J. (Engl). 128, 963-968. doi: 10.4103/0366-6999.154318

Conflict of Interest: The authors declare that the research was conducted in the absence of any commercial or financial relationships that could be construed as a potential conflict of interest.

Copyright (๑) 2020 Gong, Xu, Xu, Ding and Guo. This is an open-access article distributed under the terms of the Creative Commons Attribution License (CC BY). The use, distribution or reproduction in other forums is permitted, provided the original author(s) and the copyright owner(s) are credited and that the original publication in this journal is cited, in accordance with accepted academic practice. No use, distribution or reproduction is permitted which does not comply with these terms. 